CERN-PPE $/ 96-27$

27 February 1996

\title{
The CPLEAR Detector at CERN
}

\section{The CPLEAR Collaboration}

R. Adler ${ }^{2}$, W. Albrecht ${ }^{4}$, T. Alhalel ${ }^{11}$, A. Angelopoulos ${ }^{1}$, A. Apostolakis ${ }^{1}$, E. Aslanides ${ }^{11}$, G. Backenstoss ${ }^{2}$, F. Bal ${ }^{4}$, J.P. Bard ${ }^{14}$, D. Barraca ${ }^{14}$, C.P. Bee ${ }^{9}$, O. Behnke ${ }^{17}$, A. Benelli ${ }^{9}$, J. Bennet ${ }^{9}$, V. Bertin ${ }^{11}$, F. Blanc ${ }^{7,13}$, P. Bloch ${ }^{4}$, M. Bonnet ${ }^{4}$, C. Bula ${ }^{13}$, A. Calzas $^{11}$, P. Carlson ${ }^{15}$, M. Carroll ${ }^{9}$, J. Carvalho ${ }^{5}$, E. Cawley ${ }^{9}$, S. Charalambous ${ }^{16}$, M. Chardalas ${ }^{16}$, G. Chardin ${ }^{14}$, P. Charra ${ }^{4}$, M.B. Chertok ${ }^{3}$, A. Cody ${ }^{9}$, J. da Silva ${ }^{5}$, D. Damianoglou ${ }^{16}$, R. Daniel ${ }^{12}$, M. Danielsson ${ }^{15}$, P. Dechelette ${ }^{4}$, M. Dedieu ${ }^{12}$, S. Dedoussis ${ }^{16}$, M. Dejardin ${ }^{14}$, J. Derre ${ }^{14}$, A. Dijksman ${ }^{13}$, B. Dinkespiller ${ }^{11}$, M. Dodgson ${ }^{9}$, M. Dröge ${ }^{17}$, J. Duclos ${ }^{14}$, J. Dudragne ${ }^{4}$, D. Durand ${ }^{14}$, A. Ealet ${ }^{11}$, B. Eckart ${ }^{2}$, C. Eleftheriadis ${ }^{16}$, C. Engster ${ }^{4}$, I. Evangelou ${ }^{8}$, L. Farave1 ${ }^{7}$, P. Fassnacht ${ }^{11}$, J.L. Faure ${ }^{14}$, C. Felder ${ }^{2}$, R. Ferreira-Marques ${ }^{5}$, W. Fetscher ${ }^{17}$, M. Fidecaro ${ }^{4}$, A. Filip čič ${ }^{10}$, D. Francis ${ }^{3}$, J. Fry ${ }^{9}$, C. Fuglesang ${ }^{15}$, E. Gabathuler ${ }^{9}$, Y. Gally ${ }^{11}$, R. Gamet $^{9}$, D. Garreta ${ }^{14}$, D. Geiss ${ }^{4}$, T. Geralis ${ }^{13}$, H.-J. Gerber ${ }^{17}$, A. Go ${ }^{3,15}$, P. Gumplinger ${ }^{17}$, D. Guyon ${ }^{4}$, C. Guyot ${ }^{14}$, P. Harrison ${ }^{9}$, P.F. Harrison ${ }^{9}$, A. Haselden ${ }^{9}$, P.J. Hayman ${ }^{9}$, E. Hazen ${ }^{3}$, F. Henry-Couannier ${ }^{11}$, W.G. Heyes ${ }^{4}$, R.W. Hollander ${ }^{6}$, E. Hubert ${ }^{11}$, C. Jacobs ${ }^{4}$, K. Jansson ${ }^{15}$, H.U. Johner ${ }^{7}$, K. Jon-And ${ }^{15}$, N. Karkour ${ }^{12}$, A. Kerek ${ }^{15}$, G. Kesseler ${ }^{4}$, P.-R. Kettle ${ }^{13}$, D. King ${ }^{9}$, T. Klados ${ }^{16}$, C. Kochowski ${ }^{14}$, P. Kokkas ${ }^{2}$, K. Kontek ${ }^{13}$, R. Kreuger ${ }^{6}$, T. Lawry ${ }^{3}$, T. Lecouturier ${ }^{12}$, R. Le Gac ${ }^{11}$, F. Leimgruber ${ }^{2}$, D. Linget ${ }^{12}$, A. Liolios ${ }^{16}$, B. Lofstedt ${ }^{4}$, F. Louis ${ }^{14}$, E. Machado ${ }^{5}$, P. Maley ${ }^{9}$, U. Mall ${ }^{2}$, I. Mandić ${ }^{10}$, N. Manthos ${ }^{8}$, G. Marel ${ }^{14}$, C.-P. Marin ${ }^{4}$, H. Martin ${ }^{14}$, J.C. Michau ${ }^{14}$, M. Mikuž ${ }^{10}$, J. Miller ${ }^{3}$, F. Montanet ${ }^{11}$, T. Nakada $^{13}$, F. Nanni ${ }^{4}$, A. Onofre ${ }^{5}$, B. Pagels ${ }^{17}$, I. Papadopoulos ${ }^{16}$, P. Pavlopoulos ${ }^{2}$, F. Pelucchi ${ }^{11}$, P. Petit ${ }^{4}$, K. Philippoussis ${ }^{16}$, J. Pinto da Cunha ${ }^{5}$, A. Policarpo ${ }^{5}$, G. Polivka ${ }^{2}$, H. Postma ${ }^{6}$, Ch. Rheme ${ }^{7}$, R. Rickenbach ${ }^{2}$, B.L. Roberts ${ }^{3}$, E. Rozaki ${ }^{1}$, T. Ruf ${ }^{4}$, L. Sacks ${ }^{9}$, L. Sakeliou ${ }^{1}$, P. Sanders ${ }^{9}$, C. Santoni ${ }^{2}$, K. Sarigiannis ${ }^{1}$, M. Schäfer ${ }^{17}$, L.A. Schaller ${ }^{7}$, T. Schietinger ${ }^{2}$, A. Schopper ${ }^{4}$, P. Schune ${ }^{14}$, A. Soares ${ }^{14}$, M. Steinacher ${ }^{2}$, S. Tatsis ${ }^{1}$, L. Tauscher ${ }^{2}$, C. Thibault ${ }^{12}$, F. Touchard ${ }^{11}$, C. Touramanis ${ }^{4}$, F. Triantis ${ }^{8}$, D.A. Tröster ${ }^{2}$, I. Tsamouranis ${ }^{16}$, H. Tschopp ${ }^{7}$, P. Tsilimigras ${ }^{1}$, E. Van Beveren ${ }^{5}$, C.W.E. Van Eijk ${ }^{6}$, B. Van Koningsfeld ${ }^{4}$, J.P. Vanuxem ${ }^{4}$, G. Varner ${ }^{3}$, H.Verweij ${ }^{4}$, S. Vlachos ${ }^{2}$, D. Warner ${ }^{3}$, E. Watson ${ }^{4}$, P. Weber $^{17}$, H. Wendler ${ }^{4}$, O. Wigger ${ }^{13}$, C. Witzig ${ }^{17}$, M. Wolter ${ }^{17}$, C. Yeche ${ }^{14}$, D. Zavrtanik ${ }^{10}$ and D. Zimmerman ${ }^{3}$. 
${ }^{1}$ University of Athens, GR-10680 Athens, Greece

${ }^{2}$ University of Basle, CH-4056 Basle, Switzerland

${ }^{3}$ Boston University, Boston, MA 02215, USA

${ }^{4}$ CERN, CH-1211 Geneva 23, Switzerland

${ }^{5}$ LIP and University of Coimbra, P-3000 Coimbra, Portugal

${ }^{6}$ Delft University of Technology, 2629 JB Delft, Netherlands

${ }^{7}$ University of Fribourg, CH-1700 Fribourg, Switzerland

${ }^{8}$ University of Ioannina, GR-45110 Ioannina, Greece

${ }^{9}$ University of Liverpool, Liverpool L69 3BX, UK

${ }^{10}$ J. Stefan Inst. and Phys. Dep., University of Ljubljana, SI-61111 Ljubljana, Slovenia

${ }^{11}$ CPPM, IN2P3-CNRS et Université d'Aix-Marseille II, F-13288 Marseille, France

${ }^{12}$ CSNSM, IN2P3-CNRS, F-91405 Orsay, France

${ }^{13}$ Paul-Scherrer-Institut(PSI), CH-5232 Villigen, Switzerland

${ }^{14}$ CEA, DSM/DAPNIA, CE-Saclay, F-91191 Gif-sur-Yvette, France

${ }^{15}$ Royal Institute of Technology, KTH, S-10405 Stockholm, Sweden

${ }^{16}$ University of Thessaloniki, GR-54006 Thessaloniki, Greece

${ }^{17}$ ETH-IPP Zürich, CH-8093 Zürich, Switzerland 


\section{Abstract}

The CPLEAR collaboration has constructed a detector at CERN for an extensive programme of CP-, T- and CPT-symmetry studies using $\mathrm{K}^{0}$ and $\overline{\mathrm{K}}^{0}$ produced by the annihilation of $\overline{\mathrm{p}}$ 's in a hydrogen gas target. The $\mathrm{K}^{0}$ and $\overline{\mathrm{K}}^{0}$ are identified by their companion products of the annihilation $\mathrm{K}^{ \pm} \boldsymbol{\pi}^{\mp}$ which are tracked with multiwire proportional chambers, drift chambers and streamer tubes. Particle identification is carried out with a liquid Cherenkov detector for fast separation of pions and kaons and with scintillators which allow the measurement of time of flight and energy loss. Photons are measured with a lead/gas sampling electromagnetic calorimeter. The required antiproton annihilation modes are selected by fast online processors using the tracking chamber and particle identification information. All the detectors are mounted in a $0.44 \mathrm{~T}$ uniform field of an axial solenoid of diameter $2 \mathrm{~m}$ and length $3.6 \mathrm{~m}$ to form a magnetic spectrometer capable of full online reconstruction and selection of events. The design, operating parameters and performance of the sub-detectors are described.

The aim of the CPLEAR experiment is to study CP-, T- and CPT-symmetries in the neutral kaon system [1]. The experimental method consists of measuring asymmetries between the decay rates of $\mathrm{K}^{0}$ and $\overline{\mathrm{K}}^{0}$ into various final states $f\left(f=\pi^{+} \pi^{-}, \pi^{0} \pi^{0}, \pi^{+} \pi^{-} \pi^{0}, \pi^{0} \pi^{0} \pi^{0}, \pi \ell \nu\right)$ as a function of the decay eigentime $\tau$ :

$$
A_{f}(\tau)=\frac{\mathrm{R}\left(\overline{\mathrm{K}}^{0} \rightarrow f\right)(\tau)-\mathrm{R}\left(\mathrm{K}^{0} \rightarrow f\right)(\tau)}{\mathrm{R}\left(\overline{\mathrm{K}}^{0} \rightarrow f\right)(\tau)+\mathrm{R}\left(\mathrm{K}^{0} \rightarrow f\right)(\tau)}
$$

The experiment, installed at the Low Energy Antiproton Ring (LEAR) at CERN, uses initiallypure $\mathrm{K}^{0}$ and $\overline{\mathrm{K}}^{0}$ states produced concurrently in the annihilation channels:

$$
\begin{aligned}
& \overline{\mathrm{p}} \mathrm{p} \rightarrow \pi^{+} \mathrm{K}^{-} \mathrm{K}^{0} \\
& \overline{\mathrm{p}} \mathrm{p} \rightarrow \pi^{-} \mathrm{K}^{+} \overline{\mathrm{K}}^{0},
\end{aligned}
$$

each with a branching ratio of $\approx 0.2 \%$. The strangeness of the neutral kaon is tagged by the charge of the accompanying kaon and is therefore known event by event. The momentum of the produced $\mathrm{K}^{0}\left(\overline{\mathrm{K}}^{0}\right)$ can be obtained from measurement of the $\pi^{ \pm} \mathrm{K}^{\mp}$ pair kinematics.

The detector specifications are based on the experimental requirements, which are:

- To select reaction (1) from the (very) large number of multi-pion annihilation channels. In particular, very efficient kaon identification is essential.

- To distinguish between the various neutral kaon decay channels.

- To measure the decay eigentime between 0 and $\approx 15 \mathrm{~K}_{\mathrm{S}}$ mean lives. At the highest $\mathrm{K}^{0 a}$ ) momentum measured in our experiment $(750 \mathrm{MeV} / c)$, the $\mathrm{K}_{\mathrm{S}}$ mean decay length is $4 \mathrm{~cm}$. This sets the size of the cylindrical $\mathrm{K}^{0}$ decay volume to a radius of $\approx 60 \mathrm{~cm}$.

- To acquire a large quantity of statistics, which requires both a high rate capability $(1 \mathrm{MHz}$ annihilation rate) and large geometrical coverage.

An important aspect in the design of the experiment is the need to minimize neutral kaon regeneration effects in the decay volume by minimizing the amount of matter in the detector. The regeneration effects modify the time evolution of initial $\mathrm{K}^{0}$ and $\overline{\mathrm{K}}^{0}$ differently. The regeneration amplitude has not been measured in our $\mathrm{K}^{0}$ momentum range and must be inferred from previous measurements of charged kaon cross-sections.

Since the antiproton reaction (1) is observed at rest, the particles are produced isotropically and the detector has a typical near- $4 \pi$ geometry. The whole detector is embedded in a (3.6 $\mathrm{m}$ long, $2 \mathrm{~m}$ diameter) warm solenoidal magnet which provides a $0.44 \mathrm{~T}$ uniform field. This magnet was previously used in the DM2 $\mathrm{e}^{+} \mathrm{e}^{-}$experiment at Orsay.

The general layout of the CPLEAR experiment is shown in Fig. 1. The antiprotons are stopped in a pressurized hydrogen gas target. The use of liquid hydrogen was ruled out in order to minimize the amount of matter in the decay volume. The high pressure of the hydrogen (16 bar) and the low momentum of the incoming $\bar{p}$ beam help to keep the size of the stopping region small.

a) Unless explicitly stated, we mean by $K^{0}$ either $K^{0}$ or $\bar{K}^{0}$. 


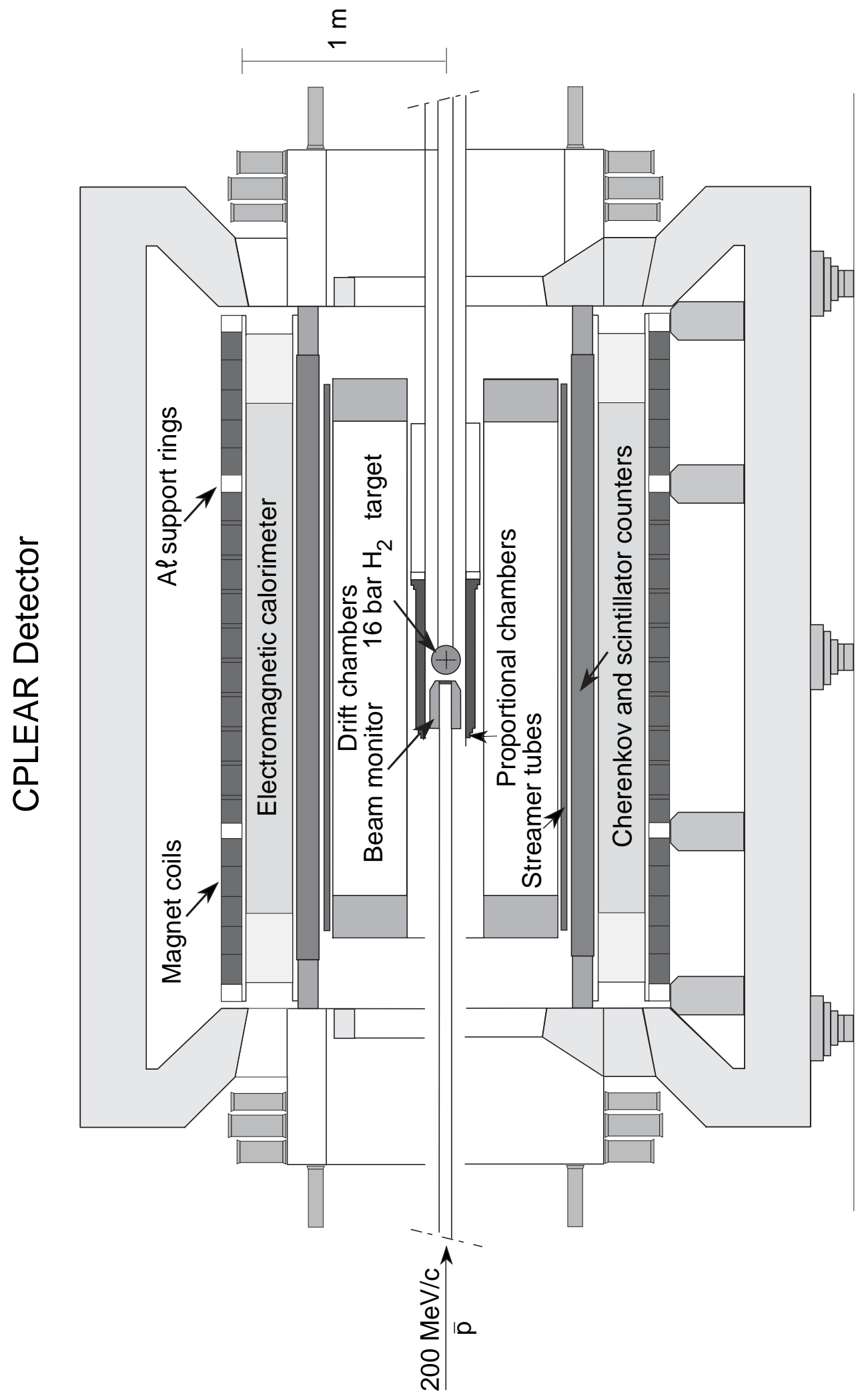

Figure 1: The general layout of the CPLEAR experiment. 
A series of cylindrical tracking detectors provides information about the trajectories of charged particles ('charged tracks' or 'tracks') in order to determine their charge signs, momenta and positions. A moderate momentum resolution $(\Delta p / p$ between 5 and $10 \%)$ is sufficient to perform the experiment. These detectors enable the annihilation vertex to be located, as well as the decay vertex if $\mathrm{K}^{0}$ decays to charged particles. To calculate the decay eigentime $\tau$, a precision of the order of a few millimetres in the vertex positions is required, but only in the transverse plane (perpendicular to the beam axis) since it can be calculated from

$$
\tau=\frac{M}{p_{T}} d_{T}
$$

where $d_{T}$ is the distance between the two vertices, $p_{T}$ the momentum of the $\mathrm{K}^{0}$, both projected onto this plane, and $M$ is the neutral kaon mass.

The tracking detectors are followed by the particle identification detector (PID), which carries out the charged kaon identification. The PID comprises a threshold Cherenkov detector, which is mainly effective for $\mathrm{K} / \pi$ separation above $350 \mathrm{MeV} / c$ momentum, and scintillators which measure the energy loss $(\mathrm{d} E / \mathrm{d} x)$ and the time of flight of charged particles. The PID is also used to separate electrons from pions below $350 \mathrm{MeV} / c$.

The outermost detector is a lead/gas sampling calorimeter used to detect the photons produced in $\pi^{0}$ decays. The design criteria of the calorimeter are mainly dictated by the required accuracy on the reconstruction of the $\mathrm{K}^{0} \rightarrow 2 \pi^{0}$ or $3 \pi^{0}$ decay vertex. The calorimeter provides $\mathrm{e} / \pi$ separation at higher momenta $(p>300 \mathrm{MeV} / c)$ and is complementary to the PID.

The small value of the branching ratio for reaction (1) and the necessary high annihilation rate place stringent requirements on the experiment. To reduce the dead time due to data acquisition and to limit the amount of recorded data, the unwanted events need to be removed efficiently. A set of hardwired processors (hereinafter referred to as HWP) has been specially designed to achieve this task. The role of the processors is to provide full event reconstruction in a few microseconds (charged-track pattern-recognition and kinematics, particle identification and shower counting in the calorimeter) with sufficient precision to allow event selection [2]. Thus the trigger rate can be kept well below $1 \mathrm{kHz}$.

The instrumentation to read-out the detectors is based on VME and FASTBUS standards, with the exception of beam monitoring and slow-control which use the G64 standard.

In this paper we present a detailed description of each subdetector used in the CPLEAR experiment. The beam and target are described in Section 2. Section 3 is devoted to the tracking devices and the global performance of these detectors for the charged particle reconstruction and event kinematics and vertex reconstruction. The particle identification detector is discussed in Section 4. The design and performance of the electromagnetic calorimeter are presented in Section 5, and the trigger processors in Section 6. In the present paper, we describe only the data acquisition system (DAQ) of the subdetectors up to the Root Read-out system (RRO) where these individual data are linked to an event-builder. The overall data acquisition system is described elsewhere [3].

\section{The Beam and Target}

\subsection{The antiproton beam and beam position monitoring}

The antiprotons in LEAR are slowed down to $200 \mathrm{MeV} / c$, cooled to give a momentum spread $\Delta p / p$ of $0.05 \%$, and then extracted over a period of $\approx 60$ minutes with a constant intensity of about $1 \mathrm{MHz}$. A refilling period of $\approx 15$ minutes then follows. The beam has horizontal and vertical emittance of $0.5 \pi$ and $2 \pi \mathrm{cm} \cdot \mathrm{mrad}$, respectively $(2 \sigma)$. The last part of the beam transport system to the CPLEAR experiment comprises 2 horizontal and 2 vertical bending magnets (dipoles) followed by a quadrupole doublet. These components are used to align and focus the beam onto the target centre.

The general arrangement of the beam monitoring and target components is shown in Fig. 2. Two orthogonal multiwire proportional chambers (MWPCs) are used as beam monitor (BM). They measure the beam shape in a plane perpendicular to the geometrical axis of the 
CPLEAR detector, in order to ensure that the beam is centred on the target window with the smallest possible spot size ( $3 \mathrm{~mm}$ FWHM).

The $\mathrm{BM}$ is positioned $35 \mathrm{~mm}$ upstream of the target entrance and is mounted together with a scintillator (beam counter) on the end of the beam pipe in a light-tight carbon-fibre enclosure. The beam leaves the beam pipe through a $100 \mu \mathrm{m}$ Be window and exits the BM housing through a $50 \mu \mathrm{m}$ aluminized-Mylar window positioned just in front of the target window. An adjustable Be degrader is incorporated in the BM housing and is used to reduce the $\bar{p}$ momentum to 80 $\mathrm{MeV} / c$ after passage through all the components, so that it can be brought to rest in the gas of the target.

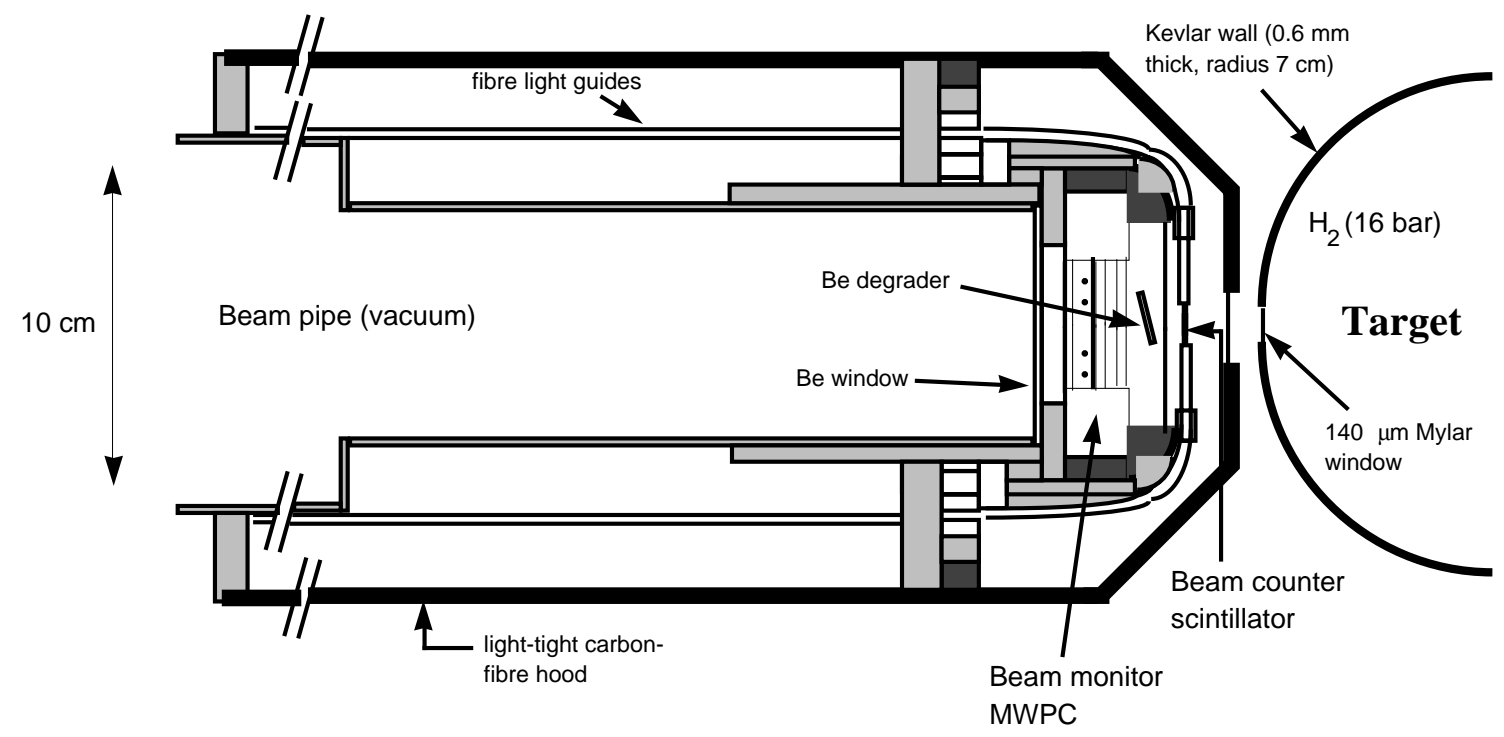

Figure 2: The beam pipe, beam monitor and target layouts.

It is important to keep the beam well-centred since the HWP processors assume that beam stops at the target centre. A beam centring system is used to automatically position the beam in the centre of the target entrance window. Otherwise, the beam might drift due to power supply instabilities in the beam line components and cause interactions in the target walls. The $\mathrm{BM}$ information is used to make small current adjustments to the horizontal and vertical dipole bending magnets in the beam line. However, due to a small misalignment of the beam line with respect to the magnetic field axis, the horizontal and vertical displacements of the beam are coupled. A correction matrix is therefore used to give the current changes.

\subsection{The beam MWPC}

The MWPCs $[4,5]$ contain $15 \mu \mathrm{m}$ diameter anode wires, $8 \mathrm{~mm}$ apart, with 8 pairs of cathode strips $(0.1 \mu \mathrm{m}$ gold on $1 \mu \mathrm{m}$ aluminium deposited on $12.5 \mu \mathrm{m}$ kapton, at right angles to the anode wires, with a pitch of $2.5 \mathrm{~mm}$ ). They have a half-gap of $2.4 \mathrm{~mm}$ and are flushed with a mixture of $60 \% \mathrm{CO}_{2}$ and $40 \%$ Ar. The low drift-velocity ensures that the displacement of the charge by the solenoid magnetic field remains small.

The MWPC cathode strip electronics consists of a 16-channel multiplexed current-tovoltage converter followed by an 8-bit ADC, which is read by the G64 DAQ system to produce horizontal and vertical projections of the beam profile. Several scans are done and a number of histograms are averaged to increase the accuracy. The $0.1 \mathrm{~mm}$ uncertainty in the mean positions of the beam centre is mainly due to digitization inaccuracy.

\subsection{The beam scintillator}

The beam scintillator (BS) is positioned just in front of the target to signal an incoming antiproton in the target. The time between scintillator passage and annihilation has an average 
value of $\approx 4 \mathrm{~ns}$, with a time spread of $\approx 2 \mathrm{~ns}(\mathrm{FWHM})$, corresponding to the stopping distribution along the beam axis. The BS signal is used as the reference time for all other detectors and starts the trigger sequence.

The BS is a $\left.\mathrm{BC} 408^{b}\right)$ disc with a diameter of $\approx 12 \mathrm{~mm}$ and a thickness of $2 \mathrm{~mm}$, mounted $10 \mathrm{~mm}$ upstream of the target window. It is inserted in a polymethyl methacrylate (PMMA) ${ }^{c}$ ) ring read by 174 light-guide fibres ${ }^{d)}$ glued to the outer circumference. The corona of fibres is bent into the upstream beam direction, grouped in two light-shielded bundles, and brought outside the solenoid magnet, where the fibres of the two bundles are read by means of a Philips XP2020 photomultiplier (PM). The PM is mounted in a Dynode Timing Base (DTB) [6] which provides an analogue output and a NIM output from a single-channel analyser. A separate lower-level discriminator is used for pile-up rejection.

\subsection{The hydrogen target}

The antiprotons are stopped in a 16-bar hydrogen-gas target, forming protonium before annihilation.

The target is spherical with an inner diameter of $140 \mathrm{~mm}$. In order to minimize the regeneration effects of the neutral kaons, the target wall is kept as thin as possible. It consists of an inner gas-tight sphere, composed of two hemispherical parts, pressed from $250 \mu \mathrm{m}$ Mylar foils in a mould at $60^{\circ} \mathrm{C}$ and glued together by an additional $120 \mu \mathrm{m}$ Mylar strip over the seam. At the top of each hemisphere the thickness of the Mylar is $140 \mu \mathrm{m}$. The Mylar is covered with an outer sphere formed of about 20 layers of Kevlar-49 fibres impregnated with Araldite. This provides the sphere with the necessary strength to withstand the high pressure, with a safety factor of 3 . The total wall thickness is $80 \mathrm{mg} / \mathrm{cm}^{2}(\approx 0.6 \mathrm{~mm})$. An area with a diameter of $13 \mathrm{~mm}$ is left uncovered for the antiproton beam entrance window. A hydrogen supply tube is connected opposite to this window to allow filling of the target.

Radiation damage tests have been performed on the window, the most fragile part of the target, but no loss in strength has been found up to $10^{6} \mathrm{~Gy}$.

The target sphere is supported at the centre of the experiment from the rear (downstream side) of the solenoid magnet and adjusted to accept the beam through the centre of the entrance window.

The slowing down of antiprotons in the combination of detectors, windows, degrading materials (including the rotatable beryllium absorber) and hydrogen gas leads to a stopping distribution well-centred inside the target along the beam axis $(F W H M=5.9 \mathrm{~cm})$ and in the perpendicular direction $(\mathrm{FWHM}=1.1 \mathrm{~cm})$.

\section{$3 \quad$ The Tracking Detectors}

The tracking detectors comprise 2 proportional chambers, 6 drift chambers and 2 layers of streamer tubes.

\subsection{The multiwire proportional chambers (PC1 and PC2)}

The two cylindrical multiwire proportional chambers (PCs) were designed to be the innermost tracking elements of the CPLEAR detector. There are two reasons for having proportional chamber information delivered rapidly to the trigger: firstly, to validate the number of tracks in the initial stages of the trigger logic; and secondly, to decide on whether or not a track is classified as a 'primary track', i.e. one which either originates from the annihilation vertex or from neutral-kaon decay at short decay time.

The cylindrical chambers contain axially placed anode wires $(15 \mu \mathrm{m}$ in diameter and with a pitch of $1.039 \mathrm{~mm}$ ) and helical cathode strips with a pitch of $2.22 \mathrm{~mm}$, giving a total of 1344 anode and 1143 cathode channels (Table 1). The chamber walls are made of a sandwich structure of Rohacell foam and Kapton foil (Fig. 3). This results in a total surface density for

\footnotetext{
b) Manufactured by Bicron Corporation, Newbury, Ohio, USA.

c) Manufactured, as well as the PMMA material of Table 3, by Röhm, Darmstadt, Germany.

d) E4001 Eska Extra, manufactured by Mitsubishi, Japan.
} 
one chamber of $41.5 \mathrm{mg} / \mathrm{cm}^{2}$, representing $1.1 \times 10^{-3}$ radiation lengths $\left(\mathrm{X}_{0}\right)$. Two wire-support rings to counteract the electrostatic repulsive forces of the charged wires are also included in the design. These rings are made of the same material as the walls, have a width of $5 \mathrm{~mm}$ and are placed some $300 \mathrm{~mm}$ apart. All 2487 channels, together with the high voltage and gas connections, are extracted from one end of the detector. The whole proportional chamber system is supported in the detector from only the downstream end of the drift chamber (DC) structure.

The chosen cell geometry, identical in both chambers, is shown in Fig. 3 and detailed information is given in Table 1 . These values, together with the choice of gas mixture (argon: $79.5 \%$, isobutane: $20 \%$, freon $13 \mathrm{~B} 1: 0.5 \%$, proportions by volume), were derived from an extensive study [7].

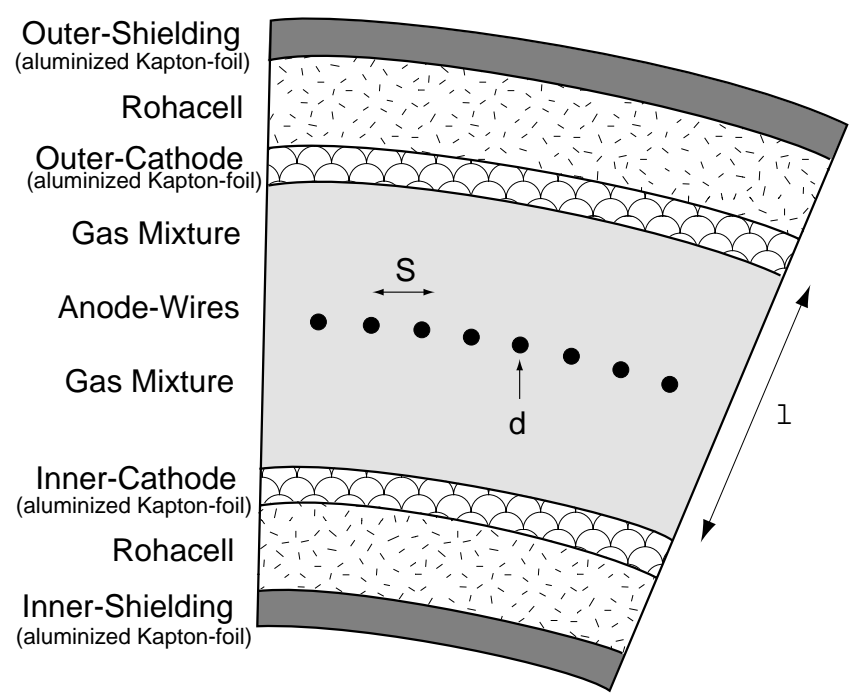

Figure 3: Section through part of a proportional chamber (not to scale).

Table 1: Proportional chamber parameters (see Fig. 3)

\begin{tabular}{|l|r|r|}
\hline \multicolumn{1}{|c|}{ MWPC Parameters } & \multicolumn{1}{c|}{ PC1 } & \multicolumn{1}{c|}{ PC2 } \\
\hline Outer radius of outer wall $(\mathrm{mm})$ & 100.25 & 132.00 \\
Radius of outer strips $(\mathrm{mm})$ & 98.25 & 130.00 \\
Radius of wire plane $(\mathrm{mm})$ & 95.25 & 127.00 \\
Radius of inner strips $(\mathrm{mm})$ & 92.25 & 124.00 \\
Inner radius of inner wall $(\mathrm{mm})$ & 90.25 & 122.00 \\
\hline Active length (mm) & 700.00 & 700.00 \\
Wall thickness (mm) & 2.00 & 2.00 \\
Full gas-gap (1) $(\mathrm{mm})$ & 6.00 & 6.00 \\
\hline Wire spacing $(\mathrm{s})(\mathrm{mm})$ & 1.039 & 1.039 \\
Number of wires & 576 & 768 \\
Wire diameter $(\mathrm{d})(\mu \mathrm{m})$ & 15 & 15 \\
\hline Strip width (mm) & 1.721 & 1.721 \\
Strip gap (mm) & 0.500 & 0.500 \\
Number of outer strips & 262 & 325 \\
Number of inner strips & 246 & 310 \\
Strip angle w.r.t. wires $(\mathrm{deg})$ & \pm 19.47 & \pm 27.89 \\
\hline Covered solid-angle $(\mathrm{sr})$ & 41.5 & 41.5 \\
Surface density of chamber $\left(\mathrm{mg} / \mathrm{cm}^{2}\right)$ & $0.963 \times 4 \pi$ & $0.939 \times 4 \pi$ \\
Radiation length equivalent $\left(\mathrm{X}_{0}\right)$ & $1.1 \times 10^{-3}$ & $1.1 \times 10^{-3}$ \\
\hline
\end{tabular}


The chamber signal processing is done in two stages (Fig. 4): the first, incorporating the preamplification and discrimination stages, is located $3 \mathrm{~m}$ away from the chambers. This electronics is identical to that used for the drift chambers, which will be described in section 3.2. It is connected by $250 \mathrm{~ns}$ delay cables to the second stage of the front-end electronics consisting of custom built cluster processors (CP300, 144 channels each) and cluster processor interfaces (CPI300, 1 per chamber), in FASTBUS crates. These modules are largely based on reprogrammable gate-arrays (Xilinx-XC2018 and Altera-EP910 chips). The gate configurations of the arrays in the Xilinx are determined via software which is downloaded from a personal computer via the CPI300. These interfaces are linked to the trigger system and the RRO system.

The input wire-hit pattern for each chamber is latched into a register by an internally generated gate signal from the CPI300, which is initiated by an external trigger signal. The masking, clustering and sectoring functions are then executed within about $170 \mathrm{~ns}$. Wire-masking can be used to suppress any hot channels. In the clustering function, adjacent wire-hits are grouped into single clusters, allowing for one eventual missing hit, and their cluster-centres are determined. In the sectoring stage, clusters are associated with a given tracking chamber element (one of 64 mini-sectors in $r \phi$ ). This coarse segmented mini-sector information is sent to the trigger from where a request can be sent back for detailed cluster-centre information on a full resolution 'wire-level'. Depending on the validity of an event, after full kinematical reconstruction a strobe signal is sent by the trigger, causing the wire addresses to be transferred to the Root Read-out system.

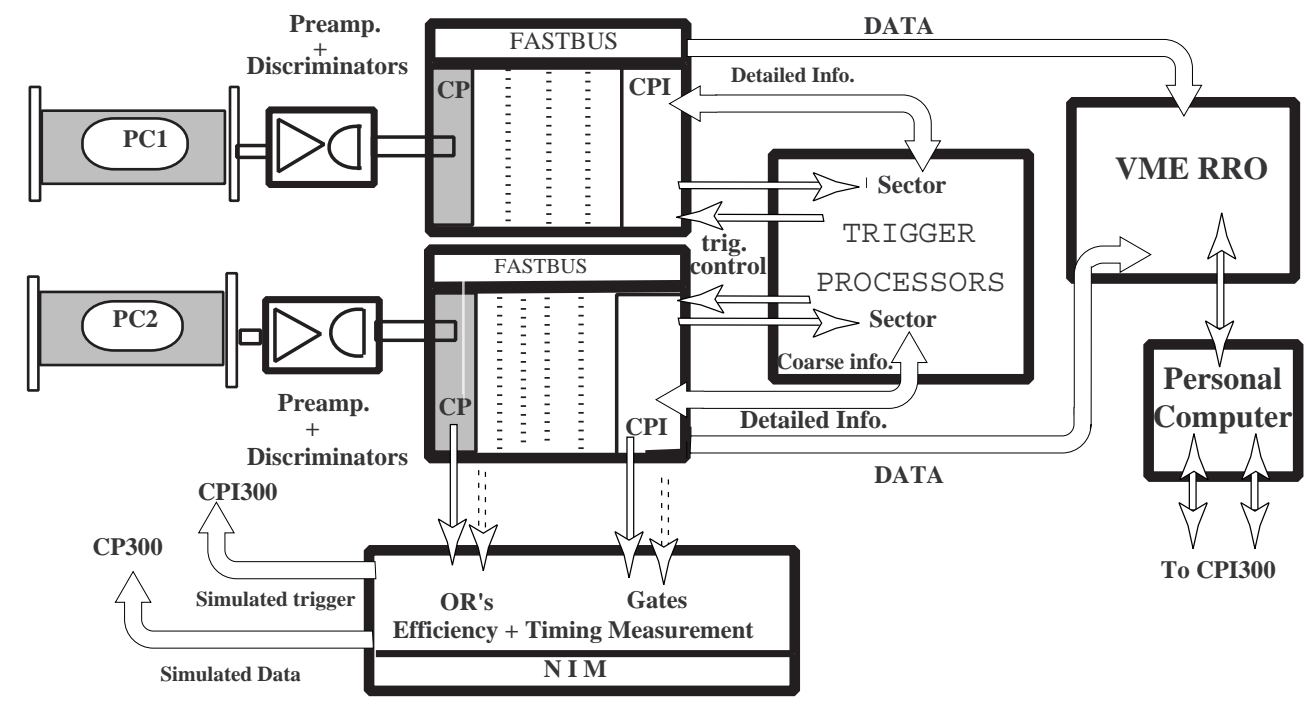

Figure 4: Block diagram of proportional chamber read-out and monitoring electronics.

A system based on fast NIM-electronics is used for testing the whole read-out chain as well as adjusting the timing of the internally generated gate signal, used for latching the raw wire hits into the CP300 memory.

The performance figures quoted below are based on a nominal high-voltage of $2700 \mathrm{~V}$ and a discriminator threshold equivalent to $0.3 \mu \mathrm{A}$. This voltage corresponds to a charge multiplication factor of $\approx 1.2 \times 10^{5}$. As can be seen from Fig. 5 , the total current drawn by one chamber at a fixed gain or high-voltage is a linear function of the charged particle rate traversing the chamber. This linearity shows that no space-charge saturation effects occur and has been tested for antiproton annihilation rates in the target of up to $2.5 \mathrm{MHz}$, corresponding to a charged particle rate traversing the chambers of $7 \mathrm{MHz}$. This is 2.5 times higher than for the normal running conditions of the experiment.

The maximum obtainable efficiency of these chambers is $98 \%$, which is due to the two wiresupport rings. The measured efficiency averaged over the whole chamber is about $97 \%$ for the nominal voltage and discriminator threshold used. This leads to a proportional chamber efficiency 
of $99.9 \%$ at the trigger-level, where a logical-'OR' is required between the two chambers. The full plateau efficiency characteristics of a prototype chamber, measured with electrons from a collimated ${ }^{90} \mathrm{Sr}$ source are shown in Fig. 6 . These characteristics have been verified with the final chambers under beam conditions, but not over the full range of high-voltages. The measured spatial resolution from reconstructed tracks, averaged over the whole chamber, is $340 \mu \mathrm{m}$, which is in good agreement with that expected from the wire spacing and an average cluster-size of 1.17 wires.

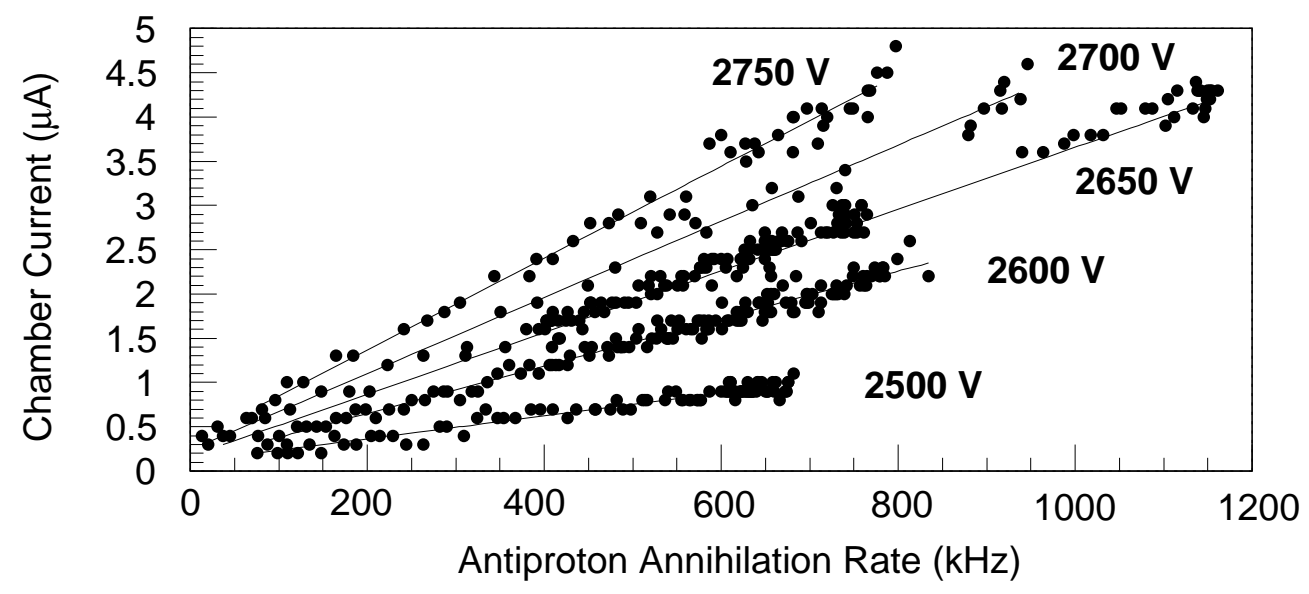

Figure 5: Plot of chamber current versus antiproton annihilation rate, taken for different highvoltage values. The plots show no space-charge saturation effects even at high rates.

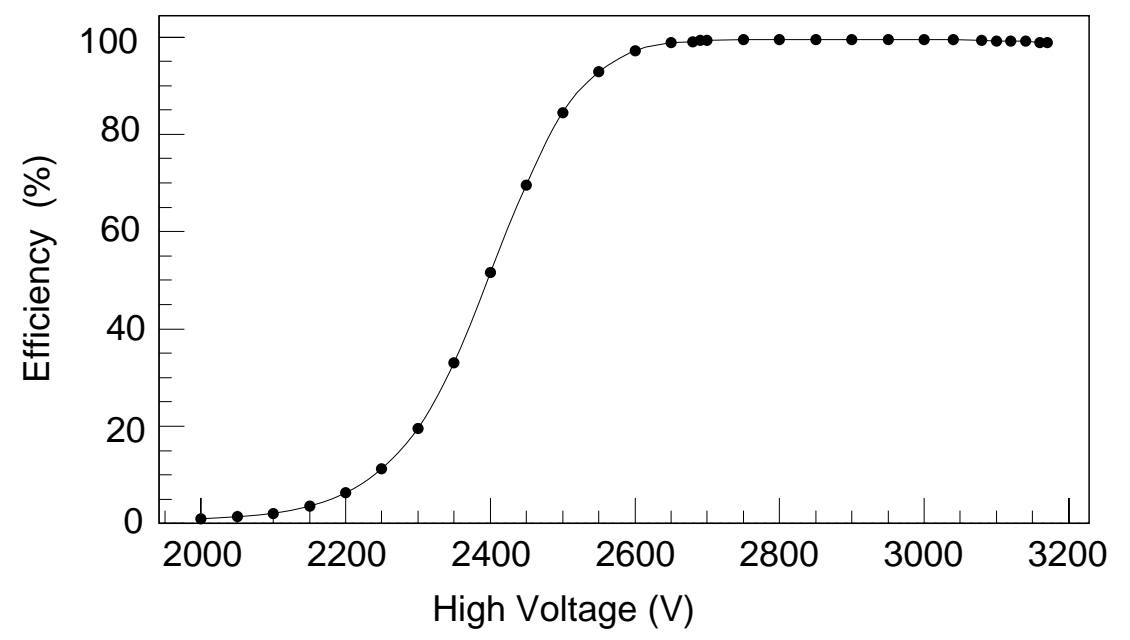

Figure 6: Efficiency curve measured with a prototype chamber and a ${ }^{90} \mathrm{Sr}$ source.

\subsection{The drift chambers (DC1 to DC6)}

Six cylindrical drift chambers (DCs) give the main tracking information $[8,9]$. The kinematic cuts calculated online by the trigger processors require a momentum resolution of $5 \%$. This is achieved by combining the measurement points in the PCs with those in the DCs (six equidistant points spread over a radial distance of $25 \mathrm{~cm}$ with a transverse spatial resolution of $\approx 300 \mu \mathrm{m}$ ). Because of the high event-rates, the drift-cell size is limited to $5 \mathrm{~mm}$ (drift time $<120 \mathrm{~ns}$ ) in order to extract the transverse tracking information for the HWP in $\approx 1 \mu \mathrm{s}$. Since the delay constraints do not allow the left/right ambiguity to be solved using software reconstruction techniques, a double sense-wire technique (separation $500 \mu \mathrm{m}$ ) is used. The drift time is digitized by a $100 \mathrm{MHz}$ clock counter giving a timing resolution $\sigma \approx 3$ ns. 
The anode wires are parallel to the beam axis and the magnetic field (Fig. 7). The electrostatic repulsion between wires at the same potential is overcome by gluing spacers made of small epoxy balls, diameter $1 \mathrm{~mm}$, positioned every $20 \mathrm{~cm}$ along the wires. (Fig. 8).

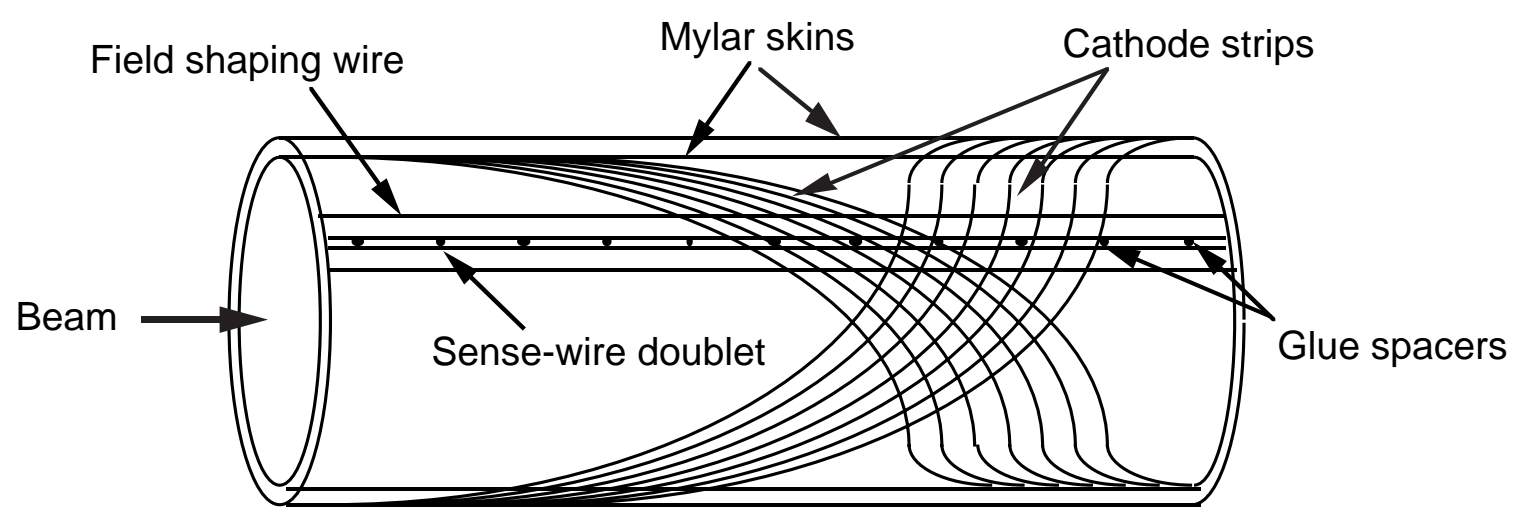

Total length $2.34 \mathrm{~m}$

Figure 7: Schematics of a drift chamber showing the sense-wire doublet and the helicoidal strips.

a)

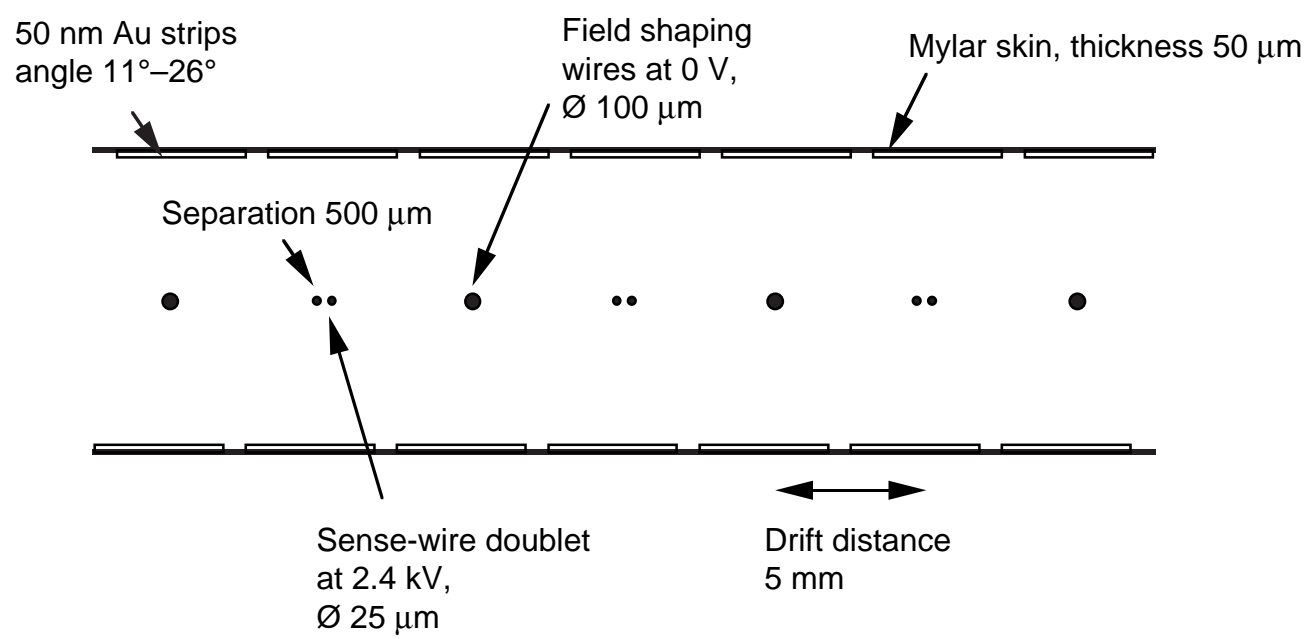

b)

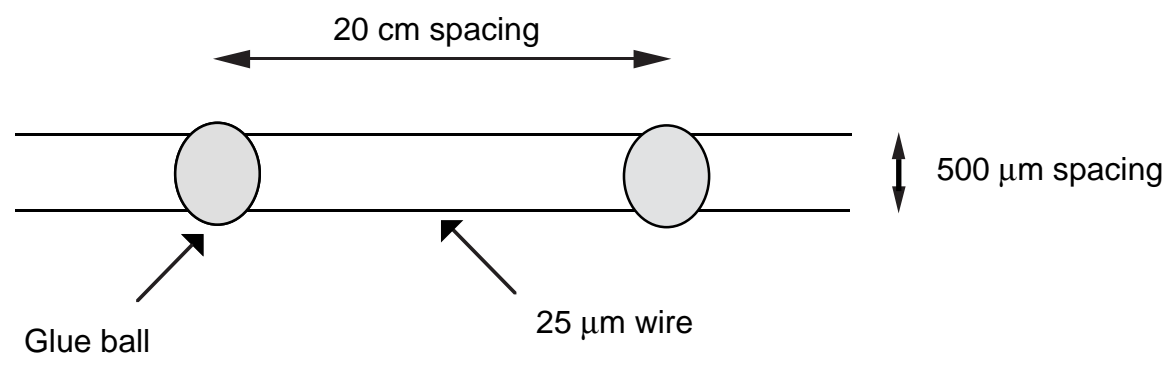

Figure 8: Detail of the drift chamber geometry: a) drift cell, b) sense-wire doublet. 
To minimize the amount of material in the tracking region, the cathode planes consist of $50 \mu \mathrm{m}$ thick Mylar membranes onto which $50 \mathrm{~nm}$ thick gold strips are deposited by evaporation. The strips are inclined at stereo angles with respect to the anode wires (Table 2) to allow the measurement of the $z$-coordinate offline by calculating the centroid of the strip charges.

Table 2: Parameters of the drift chambers

\begin{tabular}{|l|c|c|c|c|c|c|}
\hline & Radius & $\begin{array}{c}\text { Number of } \\
\text { doublets }\end{array}$ & $\begin{array}{c}\text { Number of strips } \\
\text { (inner cathode) }\end{array}$ & $\begin{array}{c}\text { Angle of } \\
\text { inner strips } \\
\text { (deg) }\end{array}$ & $\begin{array}{c}\text { Number of strips } \\
\text { (outer cathode) }\end{array}$ & $\begin{array}{c}\text { Angle of } \\
\text { outer strips } \\
\text { (deg) }\end{array}$ \\
\hline DC1 & 25.46 & 160 & 275 & 10.60 & 285 & -11.57 \\
DC2 & 30.56 & 192 & 324 & 13.06 & 336 & -15.22 \\
DC3 & 35.65 & 224 & 378 & 16.34 & 385 & -18.20 \\
DC4 & 40.75 & 256 & 424 & 20.06 & 432 & -21.00 \\
DC5 & 45.84 & 288 & 465 & 23.04 & 477 & -23.26 \\
DC6 & 50.95 & 320 & 510 & 25.40 & 520 & -25.41 \\
\hline
\end{tabular}

The gap between anodes and cathodes is maintained by exerting a differential over-pressure between two adjacent volumes as shown in Fig. 9. The pressure increases from the outer volume to the inner one, which is separated from atmospheric pressure by a cylindrical wall of $1 \mathrm{~cm}$ Rohacell foam of density $55 \mathrm{~kg} / \mathrm{m}^{3}$. The gas mixture used is $50 \%$ ethane and $50 \%$ argon by volume.

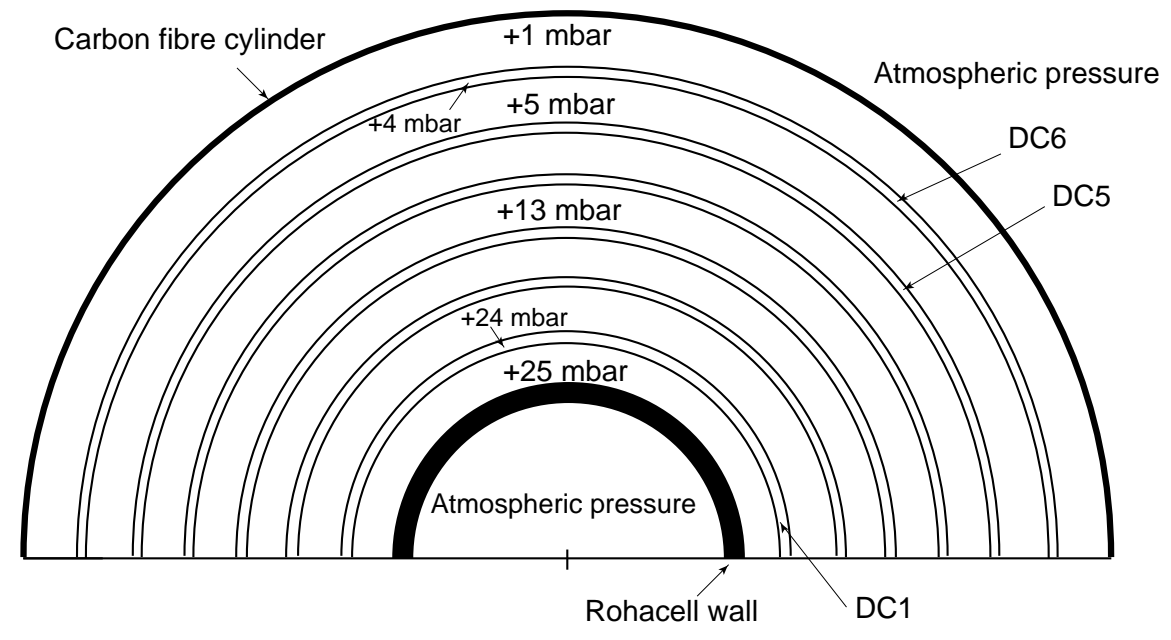

Figure 9: Pressure distribution in the drift chambers. The values shown indicate the over-pressure relative to atmospheric pressure.

The main difficulty with this type of mechanical structure is to maintain a constant gap, to within $100 \mu \mathrm{m}$ (rms), between the anode wires and the two cathode strip planes. Special tools and techniques were developed during construction to monitor this gap by measuring the reflection of a fine LASER beam from the gold strips on the cathode cylinders. Cylinders which did not fulfil the required conditions were rejected. The tension of all the wires (about 1 ton) and the stretched cathode membranes are supported by an external rigid cylinder made of a sandwich of aluminium honeycomb between two $1 \mathrm{~mm}$ thick cylinders of composite material (carbon fibre).

The total material traversed by the charged particles in the drift chambers is equivalent to $7 \times 10^{-3} \mathrm{X}_{0}$, not taking into account the outer aluminium and carbon fibre cylinder which is outside the momentum measurement volume. 
For both wires and strips, specific low-noise preamplifiers (PA) have been developed ( $\sigma_{\text {noise }}$ $=0.3 \mu \mathrm{A}$ for the current-type PA for wires and $1 \mathrm{fC}$ for the charge-type PA for strips). For the wires, the information is digitized in less than $200 \mathrm{~ns}$ using custom-made fast TDCs (Fig. 10), and is interfaced with both the Root Read-out system and the trigger system.

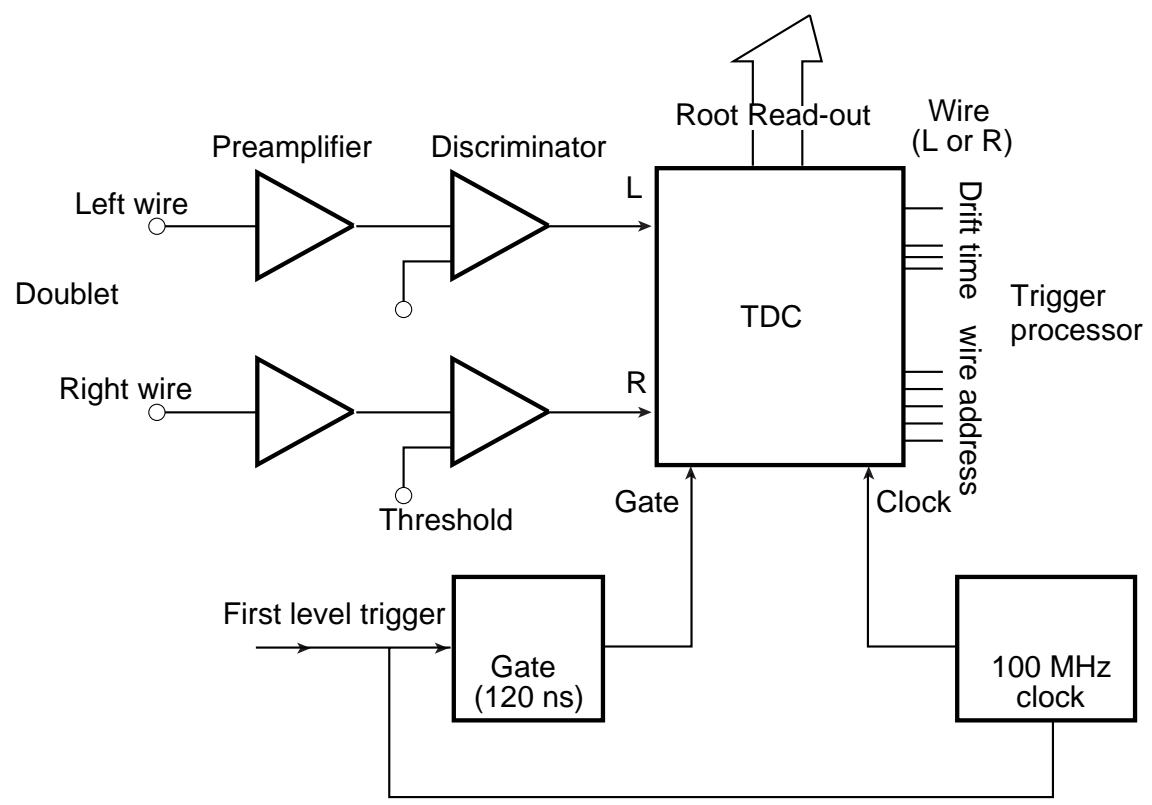

Figure 10: Block diagram of the front-end electronics for the DC wires.

The trigger processors receive the following information from the DCs:

- a bit pattern of the hit wires, necessary at the early decision level to select high- $p_{T}$ kaons;

- coarse segmented mini-sector information (where a mini-sector represents $1 / 64$ of the chamber circumference) used by the track online pattern recognition;

- on request, detailed information in a mini-sector (wire number, drift time). This information is calibrated using look-up tables to give the azimuthal coordinates of the transverse track, which are used to compute the track transverse momentum.

The performance quoted below corresponds to a voltage of $2450 \mathrm{~V}$ applied to the sense wires.

The inefficiency of the wires, with a threshold corresponding to a $0.5 \mu \mathrm{A}$ input signal to PA, is of the order of $4 \%$ (Fig. 11), mainly due to the use of the spacers between adjacent sense wires. There is also a drop in efficiency in a region $800 \mu \mathrm{m}$ (FWHM) around the doublet due to the low electric field between these wires, which are at the same potential.

The $r \phi$ resolution in the transverse plane $\mathrm{e}^{a}$ is $\approx 300 \mu \mathrm{m}$ (Fig. 12), but a deterioration of this resolution for tracks crossing the chamber can be seen close to the sense wire at the centre of the drift cell. This is due to the distortion of the electric field around the doublet mentioned above, which produces a lower global amplification. As a consequence, a significant time slewing (of the order of $10 \mathrm{~ns}$ ) increases the drift time in this region, which affects the error in the spatial measurement. This effect could be reduced, together with the inefficiency, by increasing the voltage in the chambers and hence the gas amplification. However, increasing the voltage is rendered impossible, since some antiprotons of the beam halo annihilate on nuclei of the target wall, releasing strongly-ionizing nuclear fragments which induce a discharge of the chambers at higher electric fields.

a) We use a cylindrical coordinate system with the $z$-axis defined along the incoming antiproton beam. 


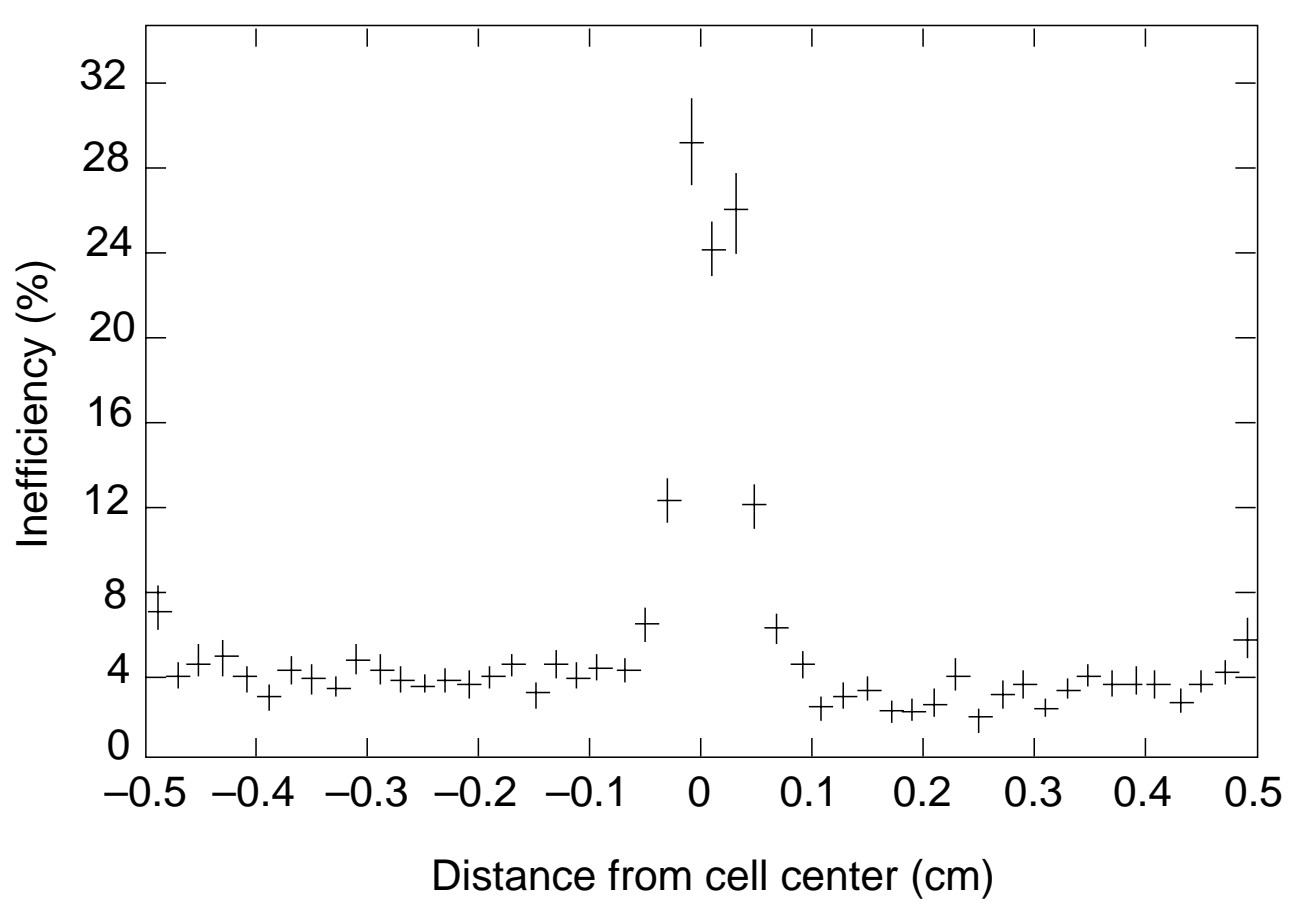

Figure 11: Wire inefficiency across the drift cell.

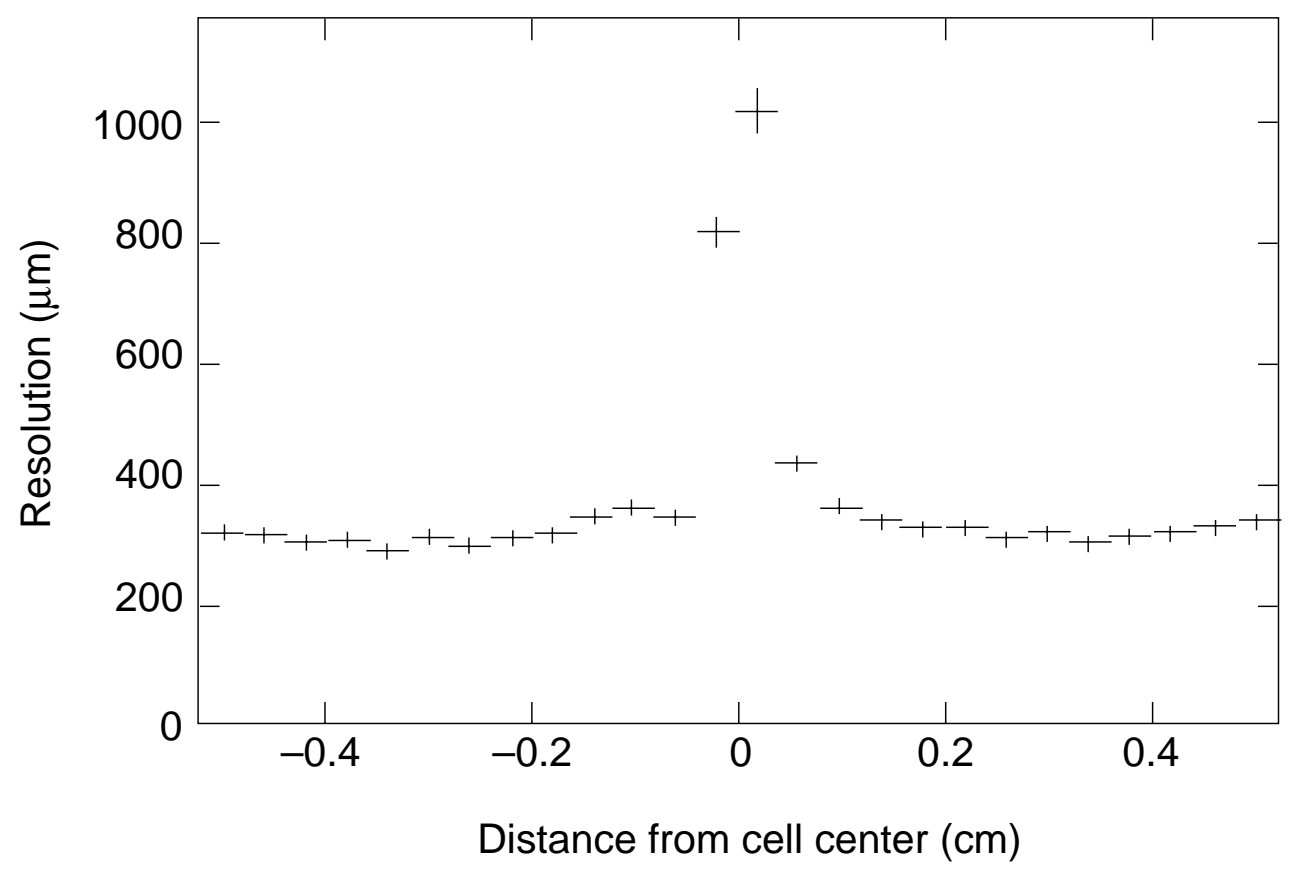

Figure 12: Spatial resolution from DC wires across the drift cell.

The strips of only four of the six drift chambers (DC1, DC2, DC4 and DC6) are equipped with read-out associated electronics, giving a total of 3106 channels. The strip charges are not used in the trigger decision (Fig. 13), and are therefore only digitized using a standard ADC (in $\approx 50 \mu \mathrm{s}$ ) if the final trigger conditions are satisfied. Only those strips with a charge above a threshold of $4 \mathrm{fC}$ after pedestal subtraction are used. This produces clusters of about 3 strips and a mean charge of $\approx 100 \mathrm{fC}$.

The strip efficiency is about $5 \%$ lower (Fig. 14) than the wire efficiency because the maximum charge induced on the strips is only one third of that induced on the anode wire. The inefficiency introduced by the presence of spacers every $20 \mathrm{~cm}$ is also clearly visible in Fig. 14 . 
(Fig. 15) to coincide with the sectors of the other detectors and to give the best hermeticity whilst allowing access for the gas supply.

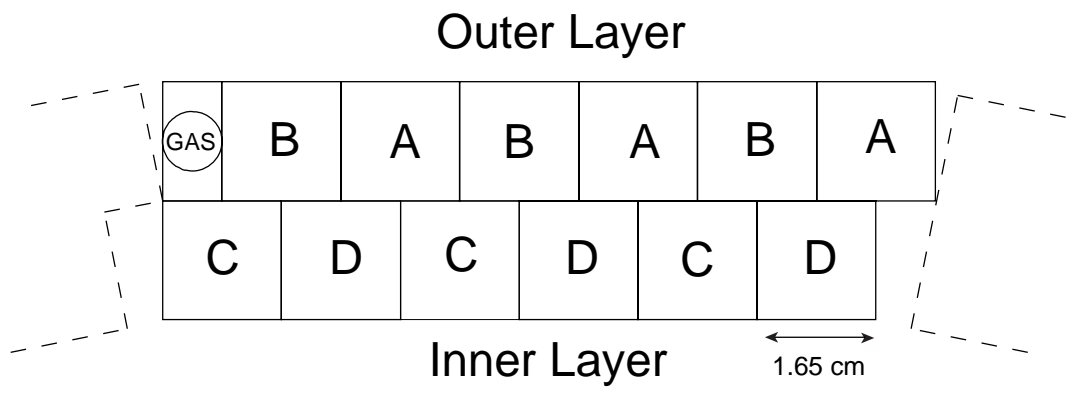

Figure 15: Layout of a streamer tube sector. A set of 3 tubes labelled with the same letter (A, B, C or D) is multiplexed to the same time digitizer.

Each tube has a central anode wire. The difference of the propagation time of the pulse along the wire to each end is used to determine the axial track position. The axial position is used by the HWP to calculate the axial momentum $p_{z}$ using the transverse momentum $p_{T}$ obtained from the DCs and assuming that the track is produced at the detector centre.

The STs are operated in the limited streamer mode characterized by large amplitude voltage pulses having fast rise times. Use of the $80 \mu \mathrm{m}$ diameter gold-plated tungsten-rhenium anode wire enables a large gain to be obtained while maintaining low wire resistance to give almost equal magnitude voltage pulses at both ends of the tube. Thus, it is unnecessary to have active electronics attached to both ends of the module.

One channel of the streamer tube electronics is shown in Fig. 16. The high-voltage feed resistor was chosen to allow extraction of $50 \mathrm{pC}$ charge at each end with a voltage drop of less than $1 \mathrm{~V}$, even at the high singles rate in each tube. The circuit provides matching of the voltage output pulses via $98 \Omega$ characteristic impedance and $6 \mathrm{~mm}$ diameter coaxial cables to the frontend electronics. It also has the facility for injection of test pulses to adjust the time-to-digital conversion (TDC). The pulse from one end of the tube is delayed by a fixed time (equal to twice the maximum transit time along the tube) so that it will always arrive after the pulse from the other end. The pulse which arrives first starts the timing process and the later pulse stops the process.

The start/stop pulses, which are typically $50 \mathrm{mV}$ in amplitude with a $5 \mathrm{~ns}$ rise time, are conveyed to leading-edge triggered discriminators in the front-end electronics, which are set to a $13 \mathrm{mV}$ threshold to reject noise. The output is a $50 \mathrm{~ns}$ wide differential ECL pulse which is delayed for trigger-timing purposes by a further $60 \mathrm{~m}$ of delay cable before entering the TDC.

The fast ECL start/stop pulses from three alternate tubes (as shown in Fig. 15) are processed into a logical hit for each tube and, taking advantage of the low rate and high conversion speed, are connected to the 3-channel multiplexed TDC measuring system based on an 8-bit flash ADC (Thomson TS8308), Fig. 17. A customized gate-array processes the start and stop pulses to check whether they originate from the same tube, and forms a map of valid hits. The TDC has a sensitivity of 1 bit per $100 \mathrm{ps}$ and the dynamic range of a tube is $\approx 200$ bits $(20 \mathrm{~ns})$. The TDC is gated by the signal from the trigger Early Decision Logic (EDL).

The total time between the passage of a track and the point where the TDC information is available in the HWP is $800 \mathrm{~ns}$, made up principally of drift time ( $\leq 200 \mathrm{~ns}$ ), delay cable $(300 \mathrm{~ns})$, and TDC and gate-array conversion time (300 ns) [10]. A further $60 \mathrm{~ns}$ is required for conversion of the TDC value to a $z$-coordinate by the HWP.

The 4 TDCs which process the hits in one complete sector are interfaced with the Root Read-out system and the trigger HWP. The entire instrumentation system is tested and calibrated using a test pulse system which applies pulses to the ends of the tubes to create pseudostart and -stop pulses just outside the active length of each tube. 


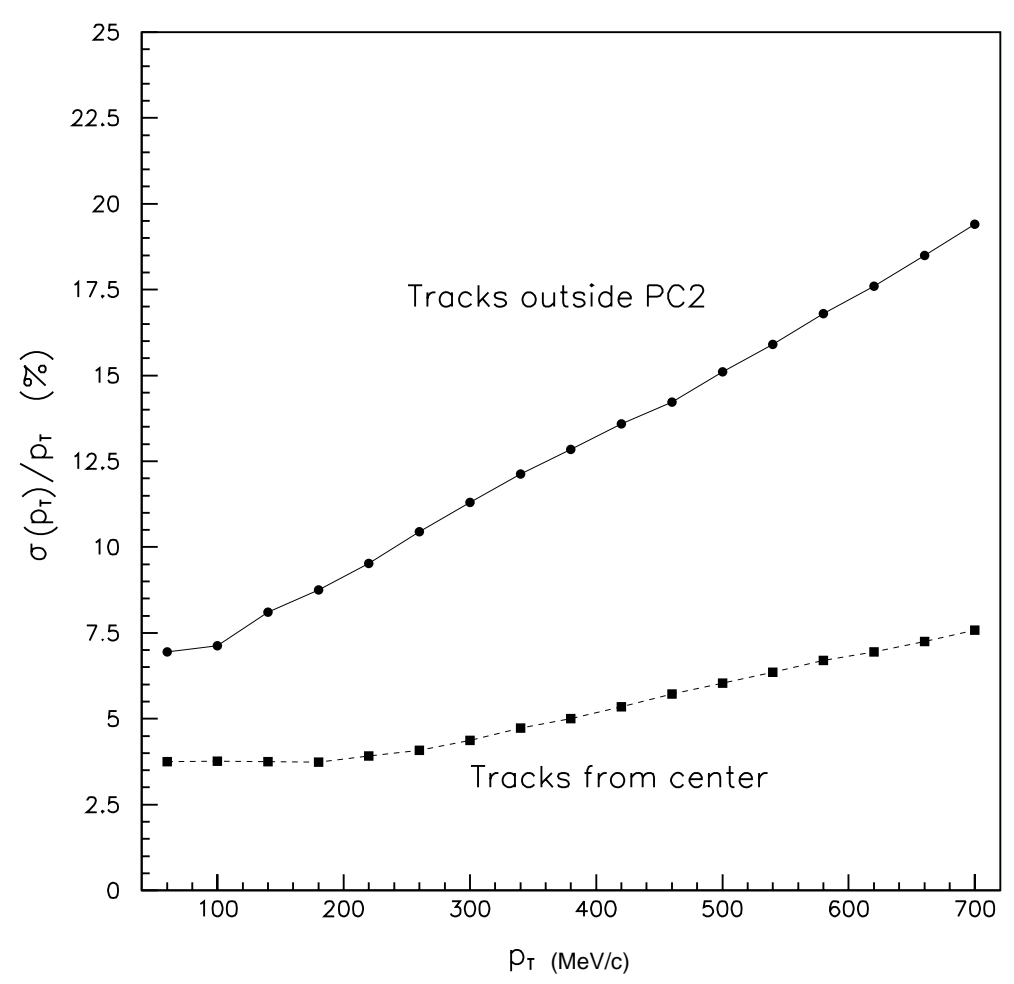

Figure 19: Relative resolution of the transverse momentum for charged tracks originating from either inside or outside the PC chambers (obtained by Monte Carlo simulated data).

a)

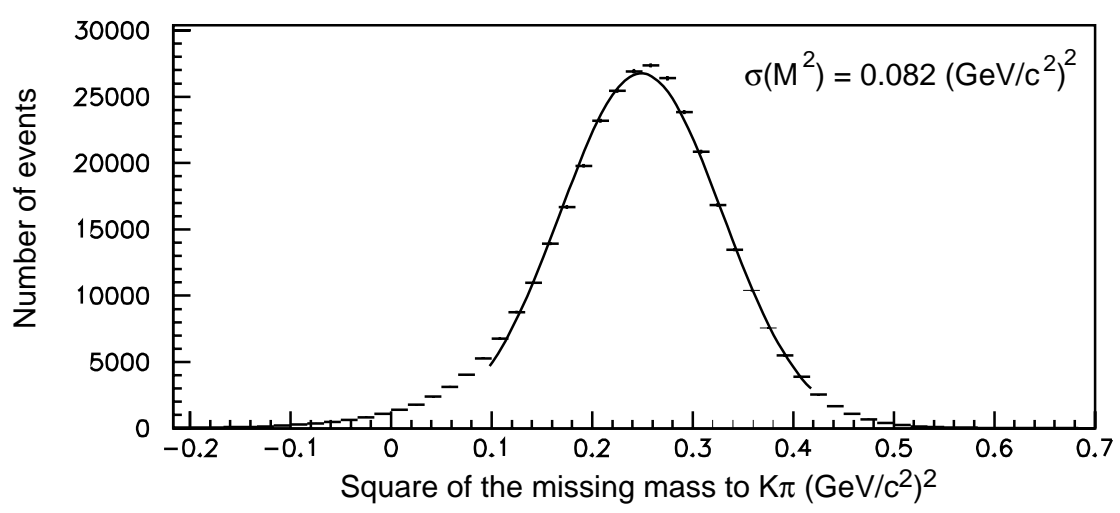

b)

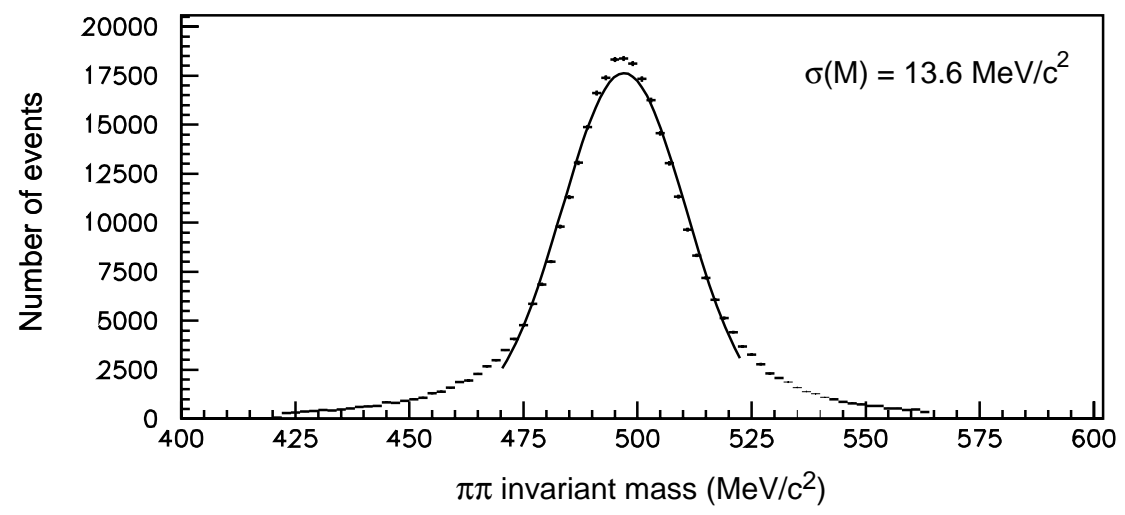

Figure 20: a) Square of the missing mass to the primary $\mathrm{K} \pi$ pair for selected $\mathrm{p} \overline{\mathrm{p}} \rightarrow \pi \mathrm{KK}^{0}$ events, and b) Invariant mass of $\pi^{+} \pi^{-}$pair for $\mathrm{K}^{0} \rightarrow \pi^{+} \pi^{-}$decays. 


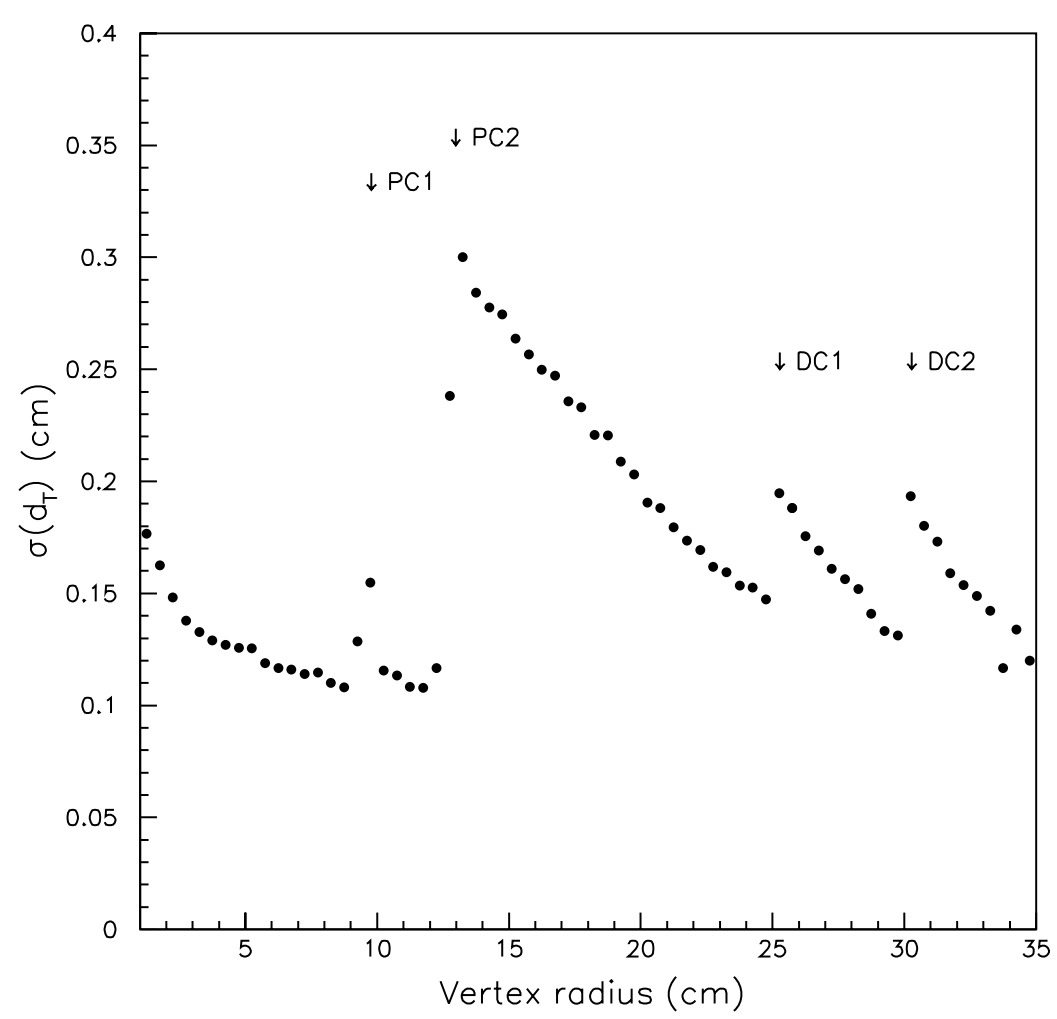

Figure 21: Resolution in the transverse distance $d_{T}$ between the annihilation vertex and the $\mathrm{K}^{0}$ decay vertex as a function of the decay vertex radius (obtained by Monte Carlo simulated data).

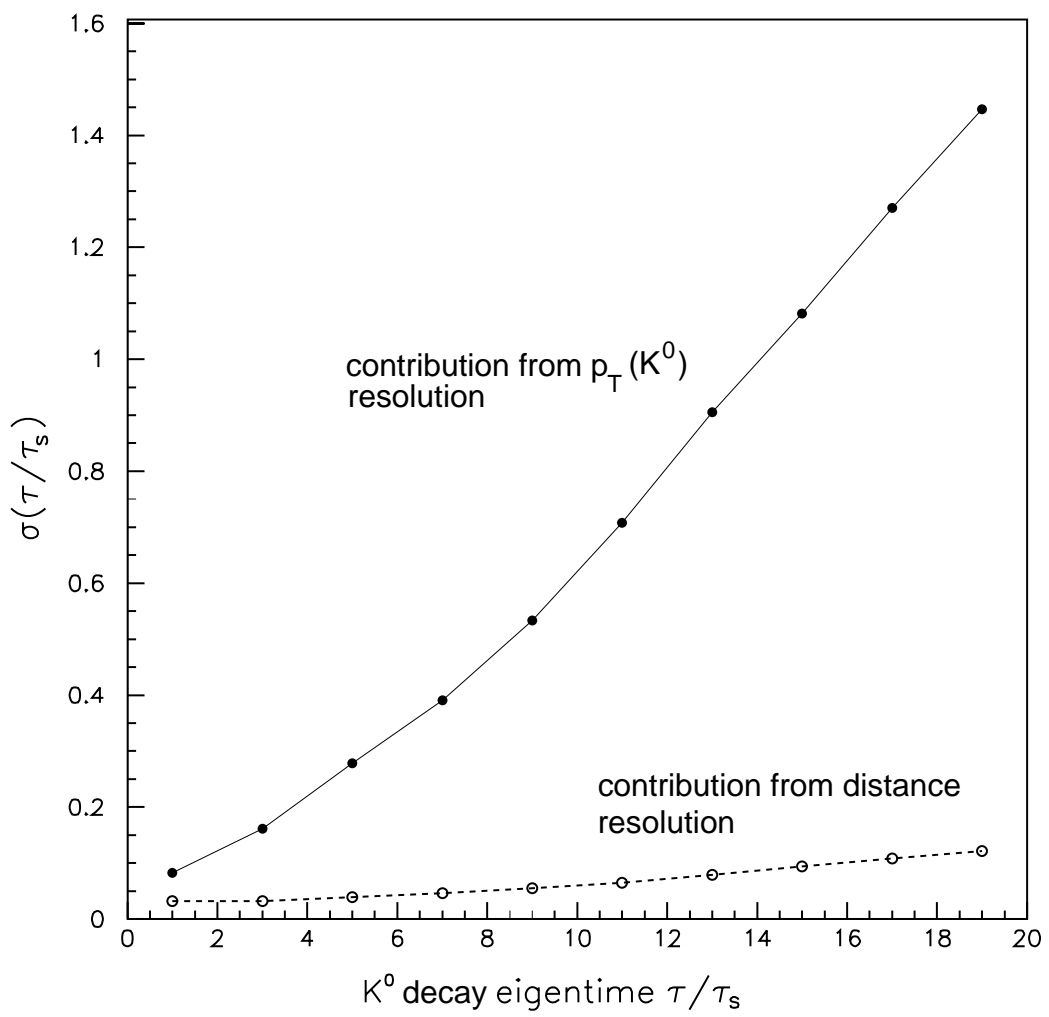

Figure 22: Decay eigentime resolution before constrained fit (obtained by Monte Carlo simulated data). The two curves show separately the contributions due to the uncertainty in the neutral kaon transverse momentum $p_{T}$ and in the distance $d_{T}$ between the annihilation and decay vertices. 
However, since the experiment studies exclusive channels, the resolution in the track parameters can be drastically improved by applying kinematically and/or geometrically constrained fits. Figure 23 illustrates the resolution obtained for the neutral kaon momentum, before and after a constrained fit, for $\mathrm{K}^{0} \rightarrow \pi^{+} \pi^{-}$events. In this particular case, a 9C fit has been applied, with 5 kinematical constraints (which require that there is conservation of energy and momentum, and that the missing mass at the annihilation vertex is the $\mathrm{K}^{0}$ mass) and 4 geometrical constraints (which require the collinearity of the $\mathrm{K}^{0}$ momentum with the line joining the 2 vertices and also require the intersection of the 2 track helices at each vertex). The $\mathrm{K}^{0}$ momentum resolution $\sigma\left(p_{T}\right) / p_{T}$ improves from $5.5 \%$ to $0.25 \%$. The decay time resolution is therefore improved, as can be seen from Fig. 24 where it is shown after a constrained fit for three different $\mathrm{K}^{0}$ decay channels.

a)
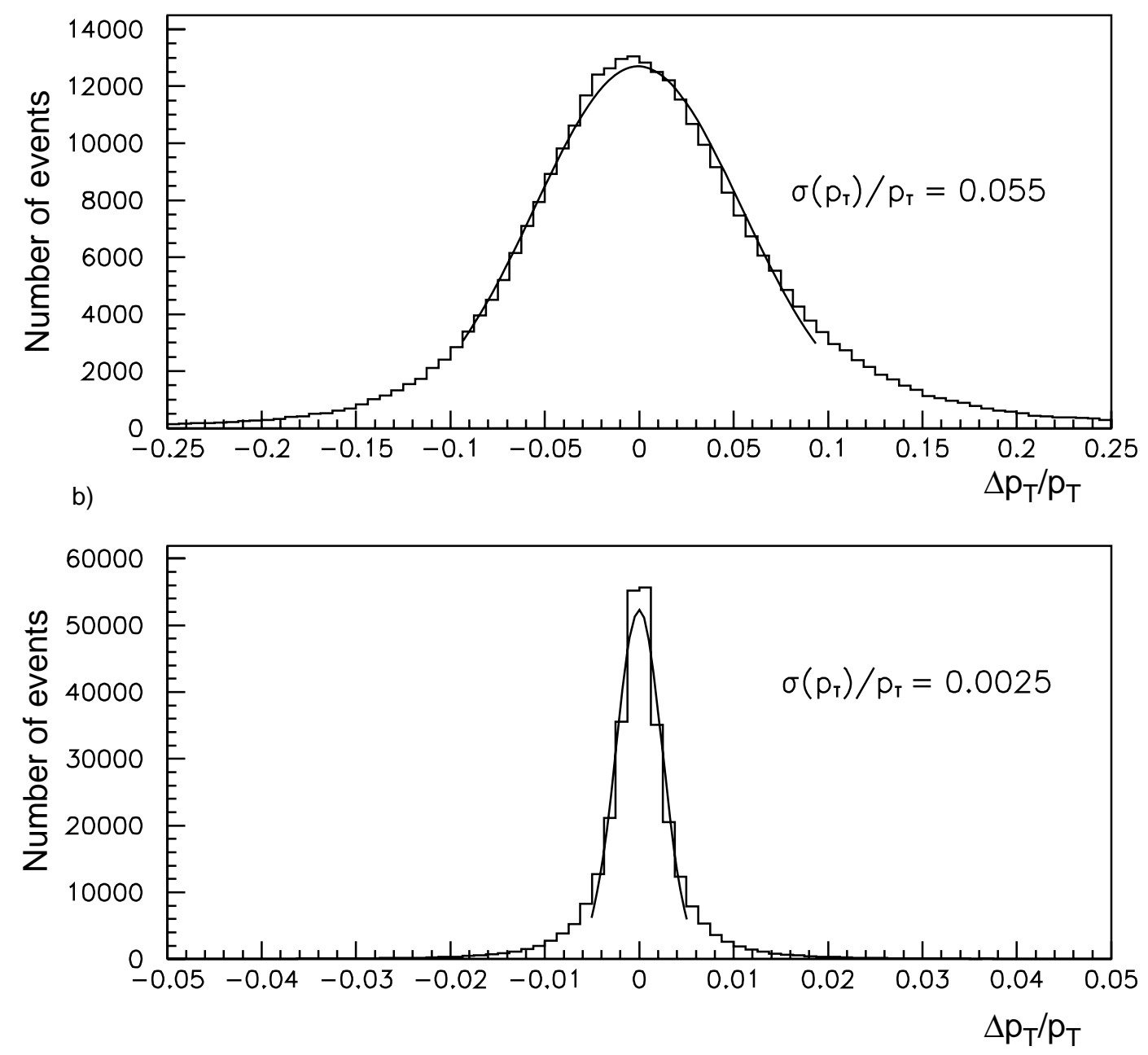

Figure 23: Distribution of $\Delta p_{T} / p_{T}$ where $\Delta p_{T}$ is the difference between the Monte Carlo generated and measured $\mathrm{K}^{0}$ transverse momentum $p_{T}$ a) before and $\mathrm{b}$ ) after constrained fit (see text).

Another important benefit of the constrained fits is that they correct for an overall momentum scale error which could result from imperfect knowledge of the magnetic field strength or from chamber misalignment. Both effects have been estimated by simulation. A $3 \%$ o shift in the value of the magnetic field produces a systematic shift in $\mathrm{K}^{0}$ decay time of $\Delta \tau / \tau<10^{-4}$, after the $9 \mathrm{C}$ fit, for $\mathrm{K}^{0} \rightarrow \pi^{+} \pi^{-}$events, and $<2 \times 10^{-4}$, after the $6 \mathrm{C}$ fit, for semi-leptonic decay events. 
at the half depth is $14 \mathrm{~cm}$. The scintillators and the Cherenkov detector are equipped with photomultipliers. At each end, the scintillators have one two-inch diameter PM while the Cherenkov has two three-inch diameter PMs for improved light guide cross-section matching and light collection efficiency. The PMs of the Cherenkov and S2 detectors are mounted perpendicular to the light guide due to the external physical space constraints of the CPLEAR detector (see Fig. 26). The PMs of the Cherenkov (Philips XP3462/PA) were chosen for their good single photo-electron resolution. The Cherenkov vessel is constructed by extrusion of UV transparent PMMA. It has a wall thickness of $2 \mathrm{~mm}$ and is closed at each end by light guides of the same material. This vessel is filled with the liquid radiator $\mathrm{C}_{6} \mathrm{~F}_{14}$ (Fluorinert FC $72^{f)}$ ) [16]. The physical characteristics of the PID are summarized in Table 3.

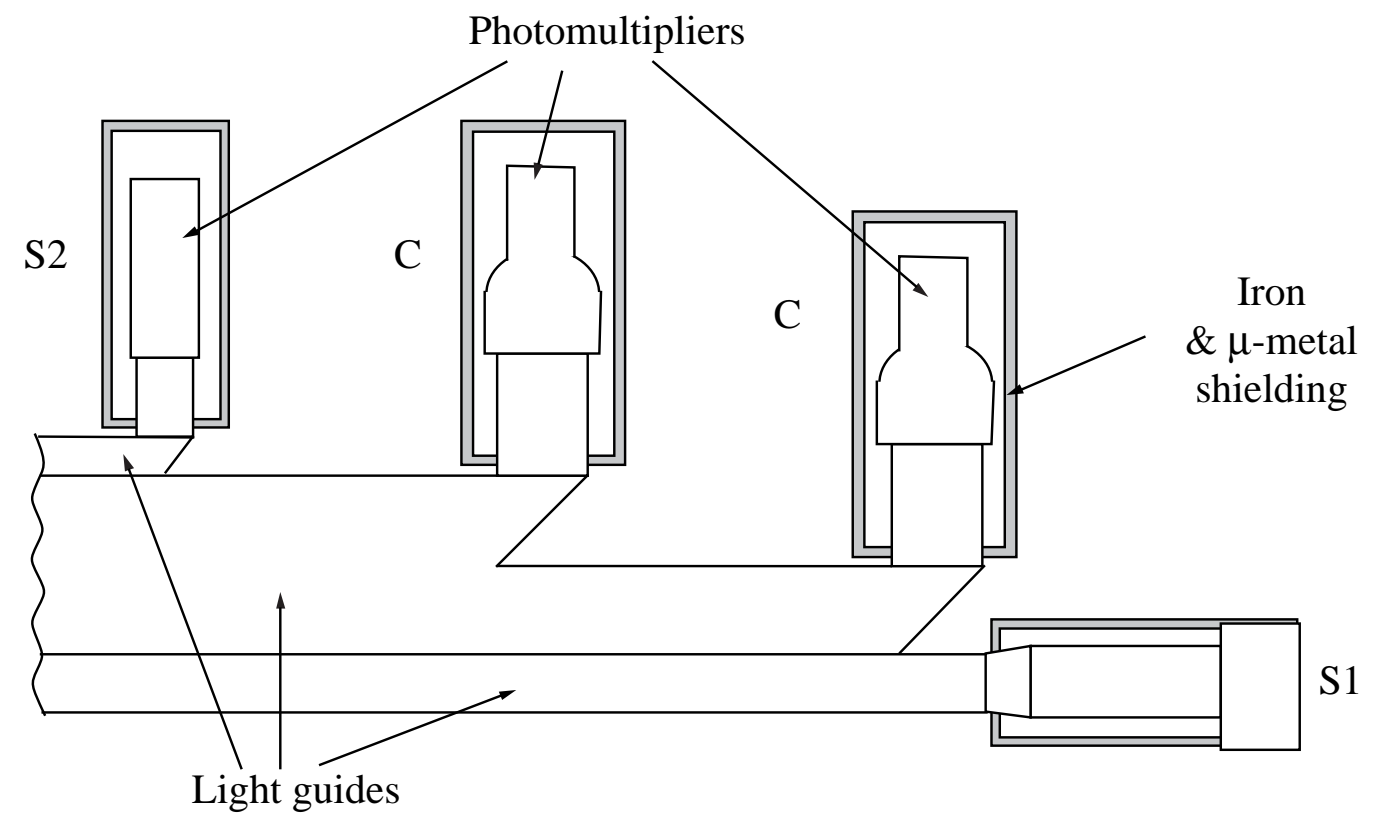

Figure 26: The photomultiplier layout for one of the 2 ends of a PID sector.

Table 3: The PID physical parameters

\begin{tabular}{|c|c|c|c|}
\hline Element & $\mathbf{S 1}$ & Cherenkov & $\mathbf{S 2}$ \\
\hline \multicolumn{4}{|c|}{ Detector } \\
\hline Material & $\overline{\mathrm{BC} 408}$ & $\begin{array}{c}\text { Extruded PMMA } \\
\text { and radiator: } \mathrm{FC} 72\left(\mathrm{C}_{6} \mathrm{~F}_{14}\right)\end{array}$ & NE $102 \mathrm{~A}$ \\
\hline \multirow{3}{*}{$\begin{array}{l}\text { Width }(\mathrm{cm}) \\
\text { Thickness }(\mathrm{cm}) \\
\text { Length }(\mathrm{cm})\end{array}$} & $11.5-12$ & $12-15.5$ & 14.5 \\
\hline & 3 & $8(7.6$ of $\mathrm{FC} 72)$ & 1.4 \\
\hline & \multicolumn{3}{|c|}{310} \\
\hline \multicolumn{4}{|c|}{ Light guide } \\
\hline \multirow{2}{*}{$\begin{array}{l}\text { Material } \\
\text { Length }(\mathrm{cm})\end{array}$} & \multicolumn{3}{|c|}{ PMMA (Plexiglas 218) } \\
\hline & 110 & 100 & 75 \\
\hline \multicolumn{4}{|c|}{ Photomultiplier } \\
\hline Type & XP4222B & XP3462 & XP2232B \\
\hline
\end{tabular}

\subsection{The Cherenkov detector}

In order not to produce any Cherenkov light by kaons of reaction (1), the Cherenkov radiator should ideally have a threshold, independent of the wavelength, of $\beta=0.84$, corresponding

\footnotetext{
f) Trademark of 3 M, St. Paul, MN, USA.
} 
to the maximum charged kaon velocity. When energy loss within the liquid radiator and the inner scintillator is taken into account this threshold is reduced to 0.81 . FC72, which has a refractive index of 1.251 at $25^{\circ} \mathrm{C}$ in the visible region of the spectrum and very low dispersion and good transparency down to wavelengths of $200 \mathrm{~nm}$, is the only liquid available in sufficient quantities which comes close to these requirements.

A specific problem is that air has a very high solubility in FC72. A dedicated system based on the use of ultrasound [17] was developed to achieve the necessary degassing.

The photo-electron yield of the radiator has been improved by the addition of $22 \mathrm{mg} / 1$ of the wavelength shifter 2,5-diphenyl Oxazole (PPO), which absorbs light between the wavelengths of 200 and $350 \mathrm{~nm}$ and re-emits in the range 300-450 nm. This high concentration of PPO was also achieved by ultrasound techniques. Measurements performed with pure FC72 as the radiator show that about half of the detected photo-electrons are due to the UV component of the Cherenkov light which has been re-emitted by the PPO.

As the kaons originate from the detector centre, there is a strong correlation between the impact point along the Cherenkov detector and the incidence angle. As a consequence, Cherenkov light cannot be extracted by total internal reflection from the central region of the detector. For this reason $\mathrm{a} \pm 40 \mathrm{~cm}$ region around the centre of the vessel is painted with a white reflective paint $\left(\mathrm{NE} 561^{g)}\right)$ to act as an isotropic diffuser. This results in a doubling of the photo-electron yield for tracks traversing this central region, significantly improving the Cherenkov light yield, so that for pions with $\beta=0.9$ the photo-electron yield per $\mathrm{PM}$ tube is everywhere $>1.8$, resulting in a total of more than 7 photo-electrons per pion.

\subsection{The scintillators ( $\mathrm{S} 1$ and $\mathrm{S} 2$ )}

The two scintillators provide the trigger signals to ensure that a charged kaon candidate traverses the Cherenkov detector. Both scintillators are laterally $1 \mathrm{~cm}$ smaller than the Cherenkov detector in such a way that tracks emerging from the centre and crossing S1 and S2 always pass through the Cherenkov's sensitive volume.

In addition to their role as a trigger hodoscope the $\mathrm{S} 1$ and $\mathrm{S} 2$ signals are used, both offline and online, to identify particles by their energy $\operatorname{loss}(\mathrm{d} E / \mathrm{d} x)$ and time of flight (TOF, S1 only).

The $\mathrm{d} E / \mathrm{d} x$ measurement is obtained from the digitized signals of each $\mathrm{PM}$, corrected for pedestal and gain. In order to be independent of the longitudinal impact point and of the attenuation length, the ionization loss is evaluated using the square root of the product of the signals measured at each end of the scintillator. A correction is applied to take into account the path length of the particle in the scintillator.

To optimize their timing characteristics, the $\mathrm{S} 1$ counters are designed to have a good light yield and a geometry which allows good detection of non-reflected photons (thus minimizing the jitter due to different light paths). The timing data from each end of S1 are added together to eliminate the propagation time in the scintillator, giving an intrinsic time resolution of $\approx 180 \mathrm{ps}$.

The time-of-flight measurement is evaluated as the TOF difference between two tracks in order to avoid the uncertainty in the annihilation time. The latter is only known with a 2 ns accuracy from the beam counter signal due to the large fluctuations in the $\bar{p}$ stopping time. The measured time difference is compared to the theoretical time difference using a particular mass hypothesis and taking into account the flight path lengths from reconstructed tracks. The effect of momentum and flight length resolutions is small compared to the intrinsic time resolutions stated above.

\subsection{The PID electronics}

The PID front-end electronics consists of three customized parts: digitization of the pulseheight information, digitization of the timing information and a crate controller which provides the interface with the trigger and Root Read-out systems.

g) Manufactured by Nuclear Enterprises Ltd., Edinburgh, UK. 
The Cherenkov and scintillator PM signals are charge-integrated within a 100 ns gate and automatically digitized at the end of the gate by 8-bit flash ADCs (Thomson TS8308) which have a digitization time of less than $100 \mathrm{~ns}$. For the S1 and S2 signals linear digitization is used. Due to the single photo-electron resolution requirements for good $\mathrm{K} / \pi$ separation and the large dynamic range required for e/ $\pi$ separation, the Cherenkov PM signals are digitized by means of bilinear digitization [18].

The $\mathrm{S} 1$ signals are processed using constant-fraction discriminators (CFD) to obtain pulseheight independent timing information. The time measured is that between the beam counter signal and the S1 signals. A custom-made analog circuit has been designed to stretch the typical timing of $50 \mathrm{~ns}$ to $300 \mathrm{~ns}$ to obtain better resolution [19]. Digitization is performed in $800 \mathrm{~ns}$ by DATEL ADCs (DATEL ADC-521, a 8-bit flash ADC followed by a 4-bit successive approximation $\mathrm{ADC}$ ).

The custom-made controller is built to handle requests for ADC and TDC data from the trigger at a rate of $25 \mathrm{MHz}$ and to handle read-out rates of 2 Mbytes/s. Fast ECL logic is used in the trigger interface section and TTL logic in the read-out stream. To reduce the read-out time, zero suppression has been implemented by the use of hit maps which are logical OR's of the $\mathrm{S} 1, \mathrm{C}$ and $\mathrm{S} 2$ signals.

\subsection{Calibration}

A real-time calibration procedure for the PID detector is needed since the PID information is used by the online trigger processors and provides most of the trigger rejection. Most of the calibration constants are measured using pionic tracks from minimum-bias events. Because of the good stability of the PID detector and its electronics, it is only necessary to perform the calibration every $\approx 24$ hours. The performance of the PID detector is permanently monitored using a subsample of physics events which is fully reconstructed and analysed by a dedicated workstation. The calibration constants consist of:

- the pedestals and minimum ionizing-particle peak positions (after correction for attenuation length) for each PM in the scintillators $\mathrm{S} 1$ and $\mathrm{S} 2$;

- the pedestals and single photo-electron peak positions, as well as the relative yield of the various PMs, for the Cherenkov detector. The latter is obtained by measuring the mean number of photo-electrons detected in each PM for energetic pions crossing the central region of the PID detector;

- the individual delays of each PM for the TOF measurement in S1.

The calibration constants are made available to the online processors by means of look-up tables which translate the raw information into physical quantities.

\subsection{PID performance}

The PID data are used online, at two stages of the trigger (see Section 6): the Early Decision Logic (EDL), based on the threshold Cherenkov response and a transverse momentum cut, defines a kaon candidate in $400 \mathrm{~ns}$; the hardwired processor HWP2 refines the kaon identification after $1.9 \mu$ s using energy loss and time-of-flight information from the scintillators.

At the EDL level, a kaon is defined by the coincidence of the two scintillator layers and the absence of any Cherenkov response $\mathrm{S} 1 \cdot \overline{\mathrm{C}} \cdot \mathrm{S} 2$ in any sector of the PID. The $\overline{\mathrm{C}}$ signal is fulfilled if not more than one PM has a signal greater than 0.5 photo-electrons. This was chosen to be a good compromise between a more stringent requirement, which would have minimized inefficiencies in the counter, and a more relaxed one which would not have removed those kaons either producing accidental background light (Cherenkov light produced in the plastic box and light from knock-on electrons) or slightly above the Cherenkov threshold. The efficiency of the $\mathrm{S} 1 \cdot \overline{\mathrm{C}} \cdot \mathrm{S} 2$ criterium has been measured at $75 \%$ for kaons with a momentum greater than $350 \mathrm{MeV} / c$. This measurement was achieved with a sample of kaons free from any trigger bias, taken from $p \bar{p} \rightarrow \mathrm{K}^{+} \mathrm{K}^{-} \pi^{+} \pi^{-}$events selected using kinematically constrained fits. The measured efficiency is shown in Fig. 27 as a function of the kaon momentum. At momenta below $350 \mathrm{MeV} / c$ 
the probability that a kaon produces an $\mathrm{S} 1 \cdot \overline{\mathrm{C}} \cdot \mathrm{S} 2$ signature drops. This is because these lowmomentum kaons do not reach S2 since their energy loss and interaction probability in S1 and $\mathrm{C}$ is higher.

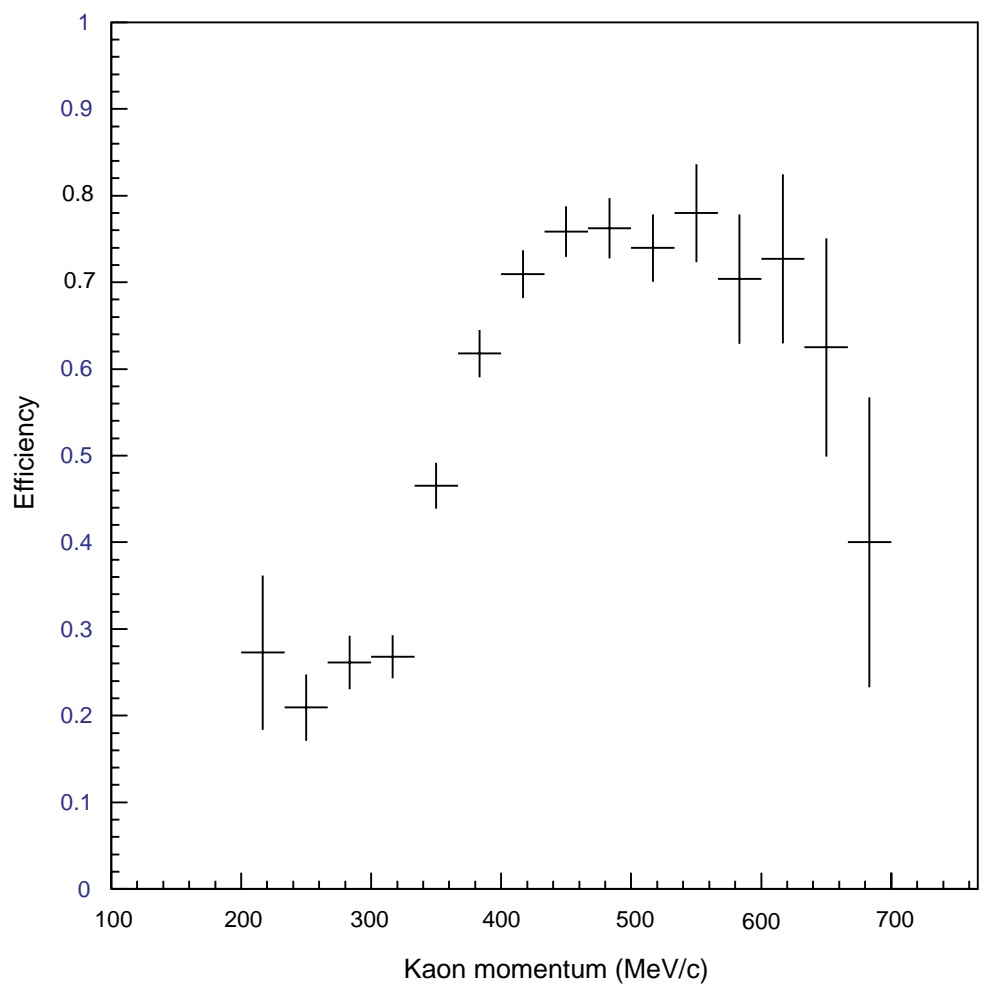

Figure 27: Kaon identification efficiency as a function of momentum, measured from $\mathrm{p} \overline{\mathrm{p}} \rightarrow$ $\mathrm{K}^{+} \mathrm{K}^{-} \pi^{+} \pi^{-}$events.

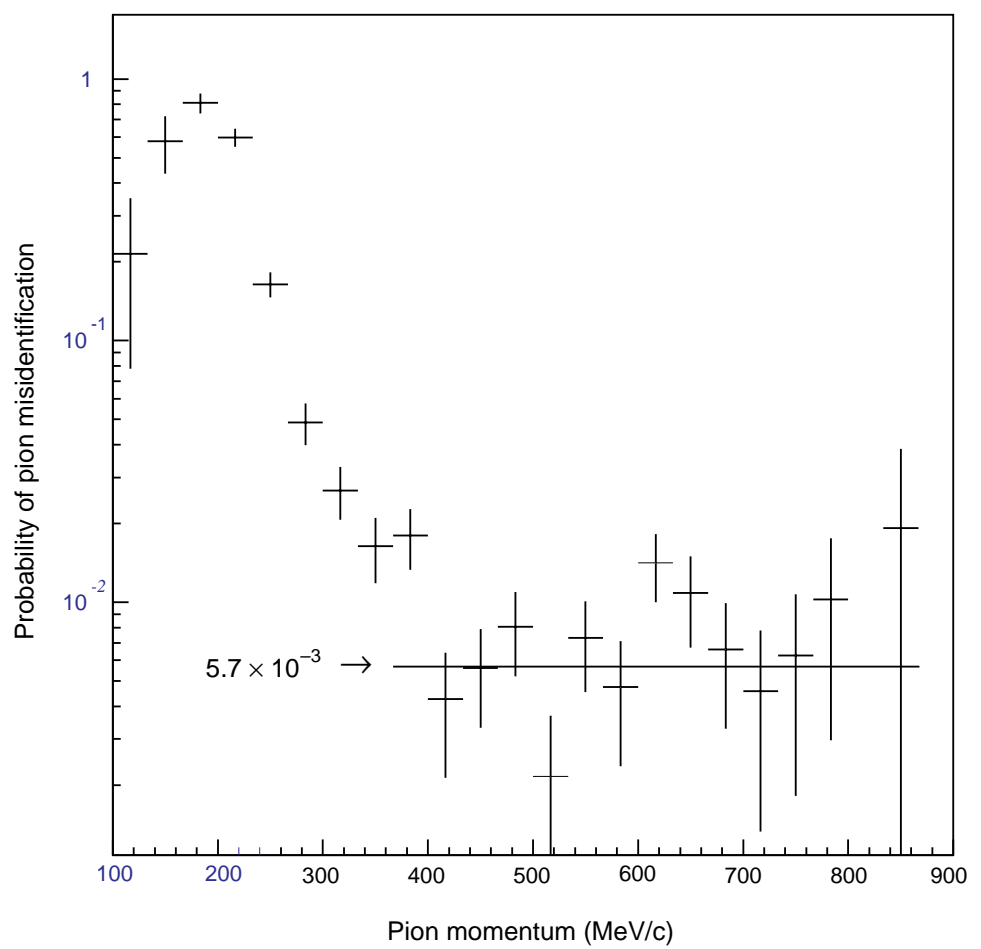

Figure 28: Probability of pion misidentification as kaon (at the EDL level). 
The pion rejection efficiency can be evaluated using well-defined $p \bar{p} \rightarrow \pi^{+} \pi^{-} \pi^{+} \pi^{-}$events selected by kinematically constrained fits. Monte Carlo studies show that the contamination of this sample by kaons is less than $6 \times 10^{-5}$. The $\mathrm{S} 1 \cdot \overline{\mathrm{C}} \cdot \mathrm{S} 2$ criterium as a function of pion momentum is shown in Fig. 28. At low momenta (below $350 \mathrm{MeV} / c$ ) the pion rejection is inefficient due to those pions which are below or just above the threshold, or which are strongly interacting in the detector material. These low momentum pions that could fake a kaon signature are removed at the EDL level by a transverse momentum $\left(p_{T}\right)$ cut, and during offline analysis by momentum cuts. This $p_{T}$ cut removes only a small fraction $(\approx 15 \%)$ of the charged kaons produced in reaction (1). For pions above $350 \mathrm{MeV} / c$, the mean probability of faking an $\mathrm{S} 1 \cdot \overline{\mathrm{C}} \cdot \mathrm{S} 2$ signature has been measured to be less than $6 \times 10^{-3}$ at EDL level, using the previous sample (Fig. 28). It should be noted that this result is not explained by statistical inefficiencies, which are calculated assuming Poisson statistics of the photo-electrons in the Cherenkov. The inefficiency is mainly due to pions interacting inside the Cherenkov or the scintillator material and leaving a $\mathrm{S} 1 \cdot \overline{\mathrm{C}} \cdot \mathrm{S} 2$ signature. This explains why the pion rejection has remained constant despite an approximate $5 \%$ per year light decrease in the Cherenkov during the 5 years of operation (due to FC72 pollution by plastics).

Figure 29 shows the $\mathrm{d} E / \mathrm{d} x$ value as a function of momentum for kaons and pions in scintillator $\mathrm{S} 1$. The resolution is better than $25 \%$ (FWHM), allowing good $\mathrm{K} / \pi$ separation up to $500 \mathrm{MeV} / c$. The $\mathrm{S} 2$ counter, being thinner has a poorer resolution of $50 \%$. The resolution in the TOF difference between the two charged tracks from the annihilation vertex is $\approx 260 \mathrm{ps}$ (Fig. 30), allowing a separation of $3 \sigma$ between $K \pi$ and $\pi \pi$ pair hypotheses for kaon transverse momenta up to $300 \mathrm{MeV} / c$, and $2 \sigma$ up to $500 \mathrm{MeV} / c$.

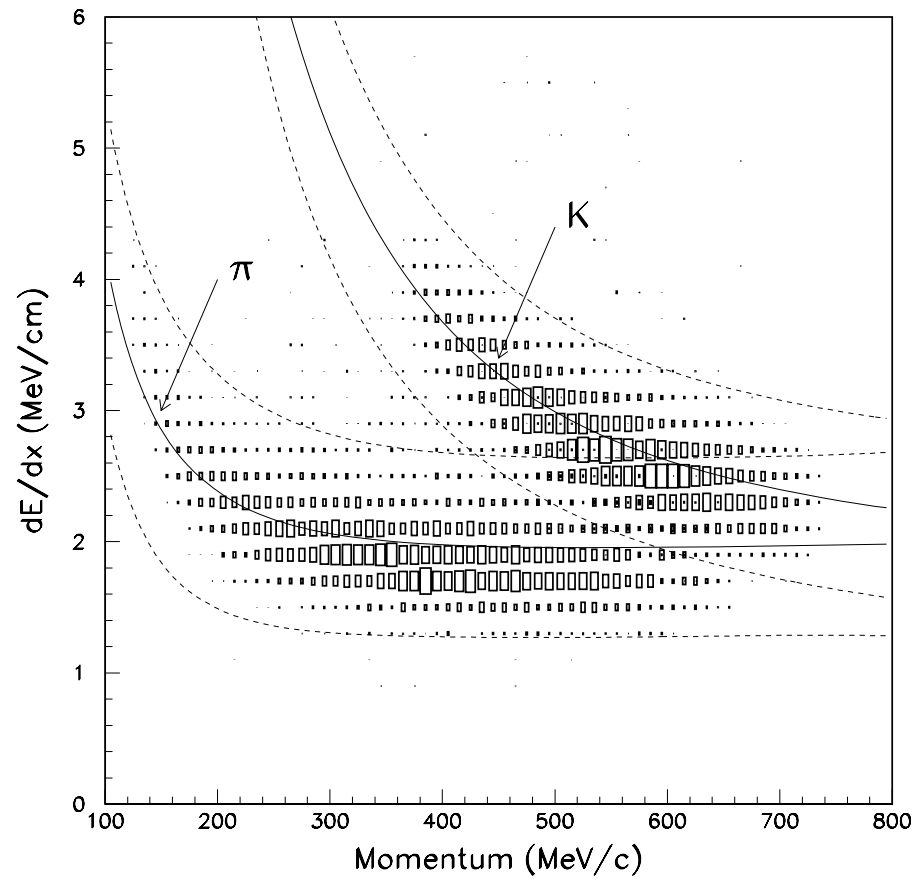

Figure 29: Energy loss measured in S1 as a function of momentum. For both kaons and pions, solid line shows the expected average energy loss and the dashed lines form a band containing $92 \%$ of the events.

The PID information is also used to perform $\mathrm{e} / \pi$ separation using neural network techniques in the momentum range $40-350 \mathrm{MeV} / c[20]$. Within the momentum range $100-200 \mathrm{MeV} / c$ an efficiency of $95 \%$ is obtained for electron recognition, which decreases to $33 \%$ at $350 \mathrm{MeV} / c$, with a constant pion background of $2 \%$ (see Fig. 31 ). 


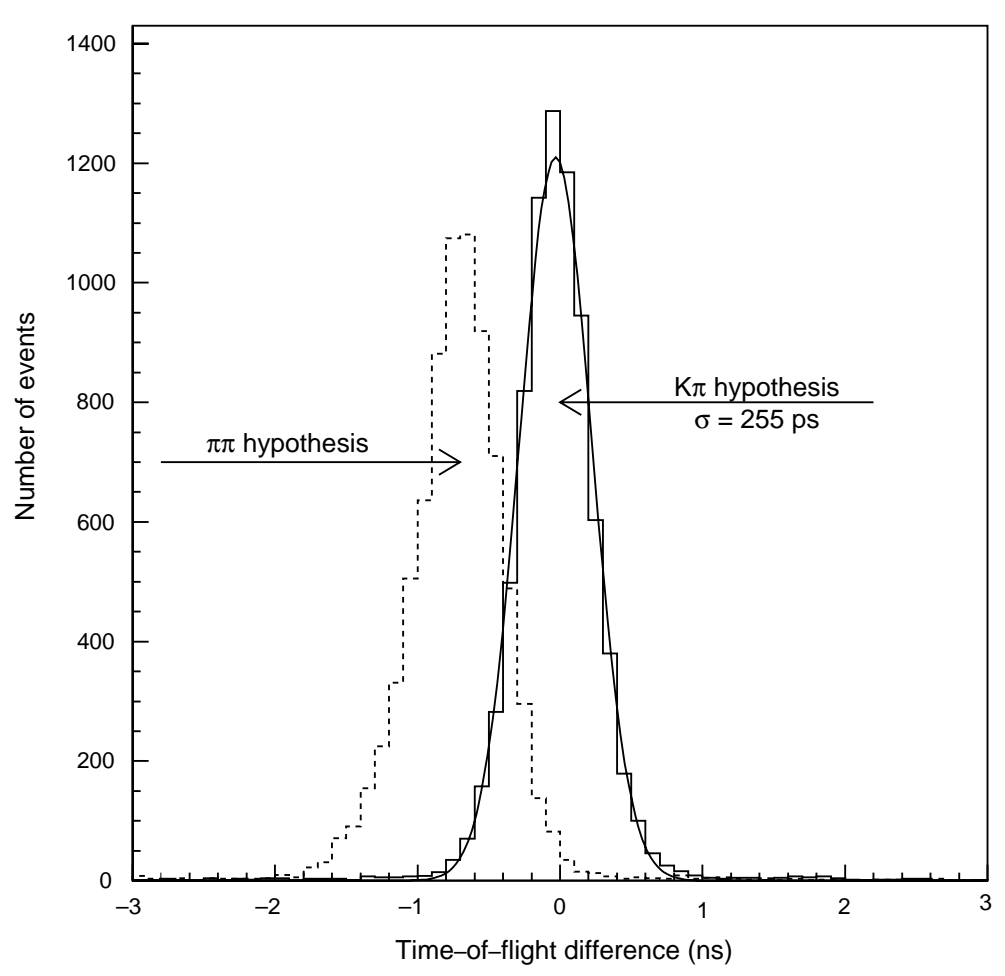

Figure 30: Time-of-flight difference between $\mathrm{K}$ and $\pi$ for the whole accepted phase space of $\mathrm{K} \pi \mathrm{K}^{0}$ events. The solid histogram corresponds to the correct particle assignment, the dashed histogram to the case where both particles are assumed to be pions.

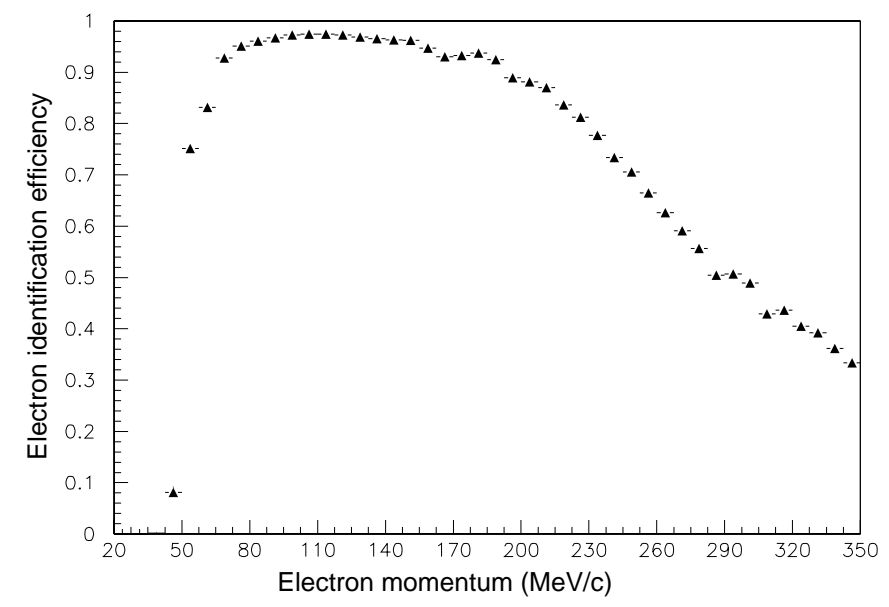

Figure 31: Electron identification efficiency from PID information as a function of the momentum.

\section{The Electromagnetic Calorimeter (ECAL)}

To study final states with neutral pions, i.e. $\mathrm{K}^{0}, \overline{\mathrm{K}}^{0} \rightarrow 2 \pi^{0}, \rightarrow 3 \pi^{0}, \rightarrow \pi^{+} \pi^{-} \pi^{0}$, we have developed a $\gamma$-ray detector, ECAL (Electromagnetic CALorimeter), with a number of novel features [21].

The ECAL is constructed in the form of a barrel without end caps (the solid angle coverage is $75 \%$ ). The barrel is an assembly of 18 lead plates alternating with sampling chambers, which consist of small cross-section tubes, and pick-up strips. The technique chosen permits a high degree of granularity in both $r$-, $r \phi$ - and $z$-coordinates, in addition to read-out flexibility. The ECAL is characterized by a total of $\approx 6 \mathrm{X}_{0}$, an energy resolution of $\sigma(E) \approx 0.13 / \sqrt{E(\mathrm{GeV})}$, and $\approx 5 \mathrm{~mm}$ position resolution for the photon conversion points. 


\subsection{Design criteria for the ECAL}

Due to the photon's low energy (50-500 MeV), a good photon detection efficiency can only be achieved with the ECAL situated inside the magnet coil, and with a fine sampling fraction. Only a moderate calorimeter thickness in terms of $\mathrm{X}_{0}$ is required.

The design studies have shown that for a $\mathrm{K}^{0}$ decaying to $2 \pi^{0}$ or $3 \pi^{0}$ the most important input in the determination of the decay vertex is the position of the $\gamma$ conversion point in the detector. A fairly poor energy resolution can be accepted provided the spatial resolution on the $\gamma$ conversion point is better than $5 \mathrm{~mm}$. However, even if it is not accurate, the energy measurement is useful for background rejection and photon pairing. The simulations have also shown that at low energy the best spatial resolution in the conversion point is obtained by sampling the shower as close as possible to the $\gamma$ conversion point. As a result, the detector demands high granularity in the $r \phi$-plane as well as good longitudinal segmentation in order to resolve the conversion points of $\gamma$ 's impinging at a small angle with respect to the detector axis.

Geometrically, the design of the ECAL was constrained from both the inner and the outer radii. The $\mathrm{K}^{0}$ decay volume, instrumented with the tracking devices and surrounded by the $12.5 \mathrm{~cm}$ thick PID, defines a minimum internal radius of $75 \mathrm{~cm}$ for the calorimeter. Externally, the ECAL had to fit within the magnet coil of $1 \mathrm{~m}$ radius.

All the previous considerations concerning geometrical constraints and optimization of performance and cost, dictated the choice of a lead/gas sampling calorimeter, rather than a crystal calorimeter such as CsI.

Small cross-section high-gain tubes were developed as the active medium [22] for the calorimeter. A prototype of ECAL, reduced in size but for number of $\mathrm{X}_{0}$, was successfully tested in a $\gamma$, e and $\pi$ beam, and the results have already been reported [23].

\subsection{Construction of the ECAL}

The ECAL consists of three $120^{\circ}$ azimuthal sectors, each one containing 18 layers identical in depth. Each layer (equivalent to $\approx \mathrm{X}_{0} / 3$ ) contains a converter plate followed by tubes of 2640 $\mathrm{mm}$ active length which are sandwiched between strip plates (Fig. 32). The converter is made of $1.5 \mathrm{~mm}$ lead sandwiched between two $300 \mu \mathrm{m}$ thick aluminium foils reinforcing the lead. The $4 \times 4.5 \mathrm{~mm}^{2}$ cross-section tubes, operated near to the limited-streamer mode, are built in the form of gas-tight packages comprising eight separate cells, hereinafter referred to as 'chambers'. There are 40-45 chambers per layer and per sector, each of $6 \times 42.5 \mathrm{~mm}^{2}$ cross-section. Both the top and bottom covers of the chambers are covered with graphite on the gas side, and on the outside they are in close contact with copper pick-up strip plates. The calorimeter contains a total of 2139 chambers (17 112 tubes or wires). The chambers are operated at $3 \mathrm{kV}$ with a gas mixture of $55 \% \mathrm{CO}_{2}$ and $45 \%$ pentane, by volume. The gas supply is serial through the chambers of a layer. The high gain of the tubes permits their read-out without amplification. The typical pulse from a wire is $20 \mathrm{mV}$ and has a width of $30 \mathrm{~ns}$ as measured for a $50 \Omega$ load.

The strips are arranged such that they cross the wires at an angle of $\pm 30^{\circ}$ allowing the reconstruction of the $z$-coordinate in each layer. The strip plates consist of a copper groundplane insulated from the copper strips which are $5.6 \mathrm{~mm}$ wide at a pitch of $6.6 \mathrm{~mm}$. The end of each strip which is not connected to the read-out is grounded via a resistor of $20 \Omega$ equal to the strip characteristic impedance. The strip signal (typically $20 \mu \mathrm{A}$ ) is fed into a current-to-voltage amplifier $(1.2 \mathrm{mV} / \mu \mathrm{A})$ in order to give a signal similar to that from the wire. There are a total of $\approx 44000$ strip channels.

The total weight of the ECAL is $\approx 8$ tons which necessitates the mechanical structure shown in Fig. 33. It consists of two $15 \mathrm{~mm}$ thick aluminium cylinders connected by $20 \mathrm{~mm}$ thick radial aluminium bars at three azimuthal angles $\left(0^{\circ}, 120^{\circ}, 240^{\circ}\right)$ to form three separate azimuthal sectors. The load of the tracking devices and the PID is suspended on the ECAL support structure. In turn, the load of this structure is distributed on four $10 \mathrm{~mm}$ thick aluminium rings interleaved with the magnet coils (Fig. 1). 
a)

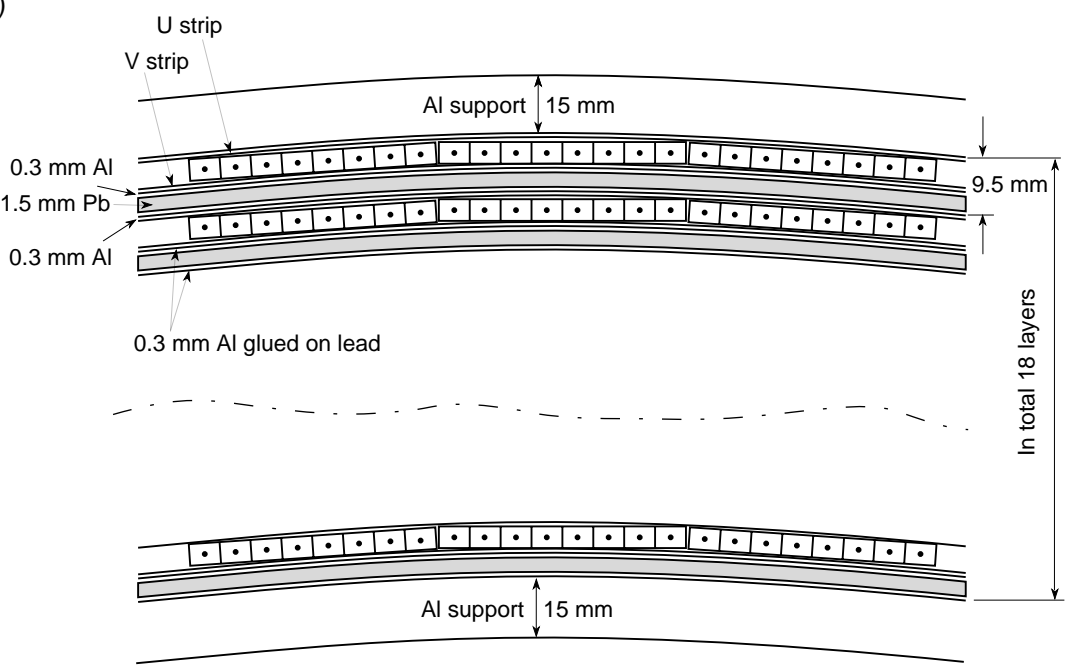

b)

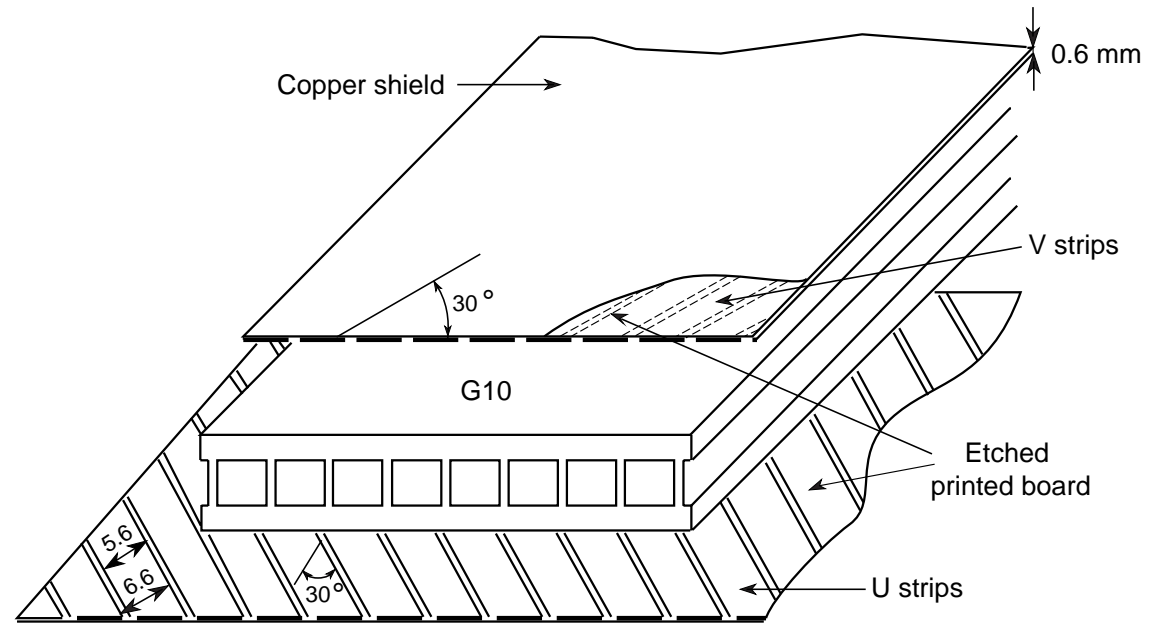

Figure 32: a) Transverse cross-section of the calorimeter. b) Geometrical set-up of the 2 layers of strips pressed on the top and bottom covers of a chamber. All lengths are in $(\mathrm{mm})$.
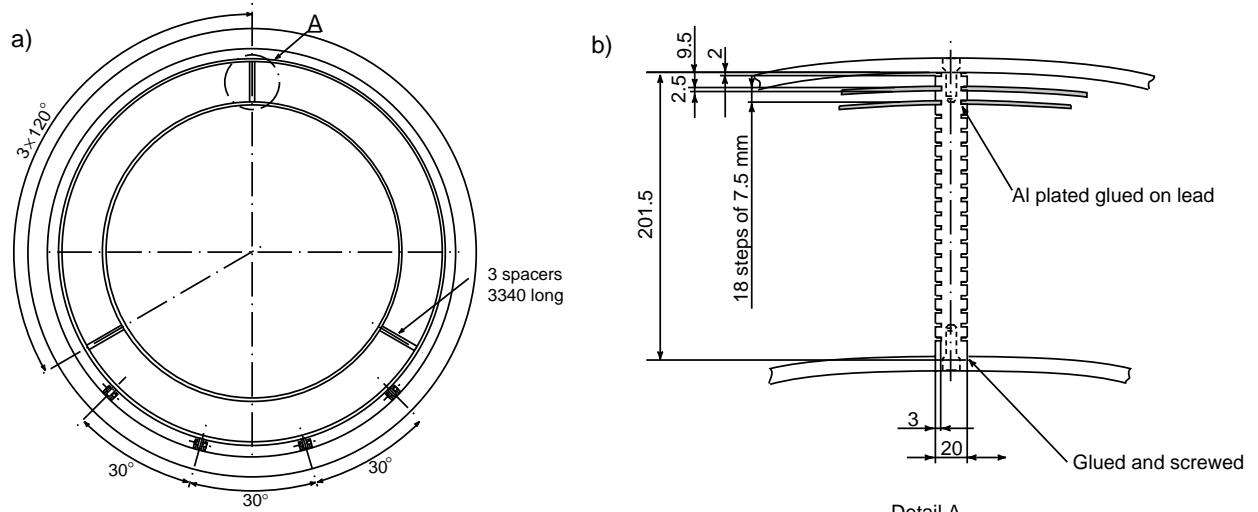

Detail A

Figure 33: a) Transverse cross-section of the mechanical support structure of the calorimeter. $b$ ) Detail of the spacer between 2 sectors. All lengths are in ( $\mathrm{mm}$ ). 


\subsection{Read-out of the ECAL}

The challenge of the calorimeter data acquisition system [24] is to provide the information of 64000 channels within a few microseconds for the second-level trigger processor and to finish the complete read-out in less than one millisecond. Modern design techniques and parallel data transfers have been used to achieve the required performance. The Fastbus standard is used throughout (Fig. 34).

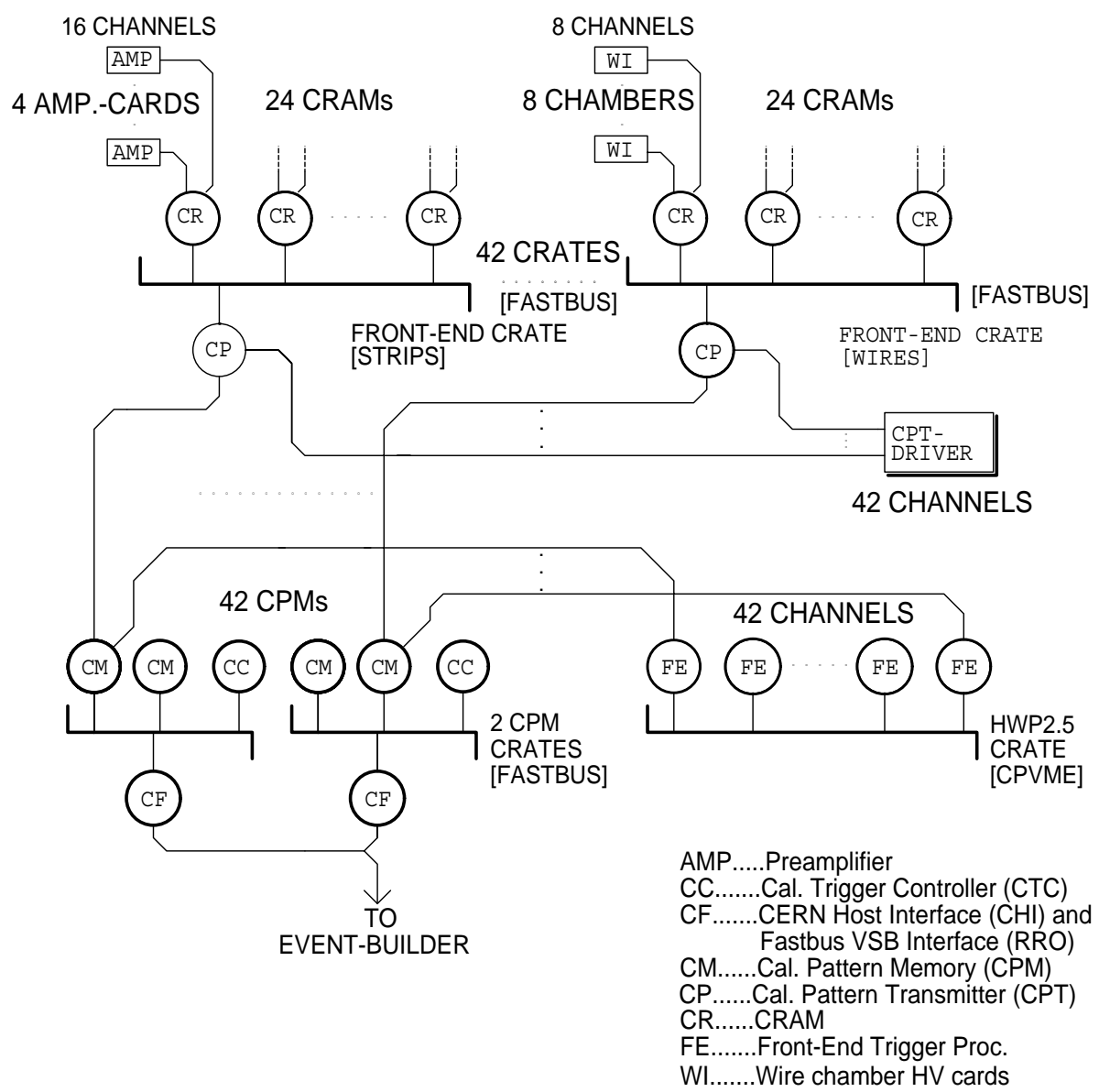

Figure 34: Block diagram of the calorimeter read-out.

The wire signals are transmitted to the front-end modules via twisted pair cables, one side of the pair being directly driven from the wire, the other terminated via a $120 \Omega$ resistor to ground. Strip signals are transmitted in differential mode from the driver of the preamplifier. A block diagram of the read-out is shown in Fig. 34 .

The wire and strip signals are received by the front-end modules (Calorimeter Receiver And Memory, CRAM). Each module contains 64 channels of input receivers, discriminators, shapers and digital delays ${ }^{h)}$. Up to 24 modules are in one Fastbus crate, thus requiring a total of 1000 modules distributed in 42 Fastbus crates for the read-out of 64000 channels. The modules run in an auto-updating mode (every $40 \mathrm{~ns}$ ) which makes resetting unnecessary. Additional features are selectable after-pulse cancellation times (set to $80 \mathrm{~ns}$ ) and selectable acceptance windows for the output register (set to $120 \mathrm{~ns}$ ). Test pulses can be sent directly to the HV boards and the inputs of the preamplifiers or the internal output registers. This allows a detailed verification of the calorimeter read-out chain and a fast check of its performance.

The information of up to 24 CRAMs is read via a special crate controller (Calorimeter Pattern Transmitter, CPT) using a daisy chain protocol. It is transferred as hit group pattern

h) Application Specific Integrated Circuit (ASIC). 
(HGP) containing the hit-map of 192 chambers and as data pattern representing the exact hit distribution of 8 chambers. This granularity enables fast online reconstruction of showers in all three projections via an external second-level trigger processor (HWP2.5, see Section 6).

The central distribution system (CPT-driver) provides signals such as clock, trigger, test pulses and discriminator thresholds via each CPT to all CRAM modules in a crate.

All 42 CPT modules are connected to 42 corresponding processing modules (Calorimeter Pattern Memory, CPM) which provide fast data collection, send the HGP information to the HWP2.5 processor and encode the data, thus minimizing the quantity of data to be recorded on tape. An average event of about 400 fired channels (out of 64000 channels in total) will give 100 32-bit words after encoding. A first-level decision starts the data transfer of all 42 CPTs in parallel ( 3 to $7 \mu \mathrm{s}$ ). The encoding of the data takes between 3.2 and $24.3 \mu$ s depending on the number of data patterns. HWP2.5 calculates in parallel the number of showers seen by the calorimeter and decides within $17 \mu$ s whether the event should be written to tape.

The final read-out is performed in parallel by two Fastbus master modules (CERN Host Interface, $\mathrm{CHI}^{i)}$ ) after the event has passed the last trigger decision. The read-out of 21 modules takes $450 \mu \mathrm{s}$ for a typical event (400 bytes). The data memory of each CHI is seen by the eventbuilder via VSB modules. A small fraction of the events (about 1 out of 100 ) is sent via Ethernet to a processor to monitor online the performance of the calorimeter.

\subsection{Performance of the ECAL}

The performance of the ECAL (the photon detection efficiency, the photon conversion position resolution, the energy resolution, and the charged particle identification) depends, for the offline analysis, on the shower pattern recognition.

A tailored shower pattern recognition algorithm searches for clusters of hits separately in the projection of the wires $(\mathrm{W})$ and the two strip planes $(\mathrm{U}, \mathrm{V})$. The minimum requirements for a $W$ projection are $\geq 3$ hits in $\geq 2$ layers, and for both $U$ and $V$ projections $\geq 2$ hits in $\geq 2$ layers. A shower is defined by the matching of $\mathrm{W}, \mathrm{U}$ and $\mathrm{V}$ projections in a single layer, taking advantage of the $\mathrm{U}, \mathrm{V}$ redundancy. The matching provides the shower conversion coordinates, i.e. the photon conversion point in the ECAL. A shower is defined as a neutral shower, if the shower conversion point is separated from any charged track, extrapolated in the magnetic field through the PID into the ECAL, by at least $25 \mathrm{~cm}$ in both the $r \phi$-plane and the $z$-direction.

The photon detection efficiency is measured with tagged photons from the neutral pion decay of the two-body annihilation reaction

$$
\mathrm{p} \overline{\mathrm{p}} \rightarrow \phi \pi^{0} \rightarrow \mathrm{K}^{+} \mathrm{K}^{-} \gamma \gamma
$$

The $\pi^{0}$ momentum vector is calculated from the $\mathrm{K}^{+} \mathrm{K}^{-}$momentum vector. With the information from one detected photon, the conversion coordinates in the ECAL can be reconstructed for the other one. If the second photon is reconstructed to be within the active volume of the ECAL, the efficiency is inferred by counting the events where the second neutral shower is found in the expected region. The photon detection efficiency as a function of the photon energy is shown in Fig. 35, reaching a plateau of $90 \%$ at a photon energy of $200 \mathrm{MeV}$. About $5 \%$ of the inefficiency is due to the support structure. The inefficiency for low-energy photons is partly due to the detector material in front of the ECAL, which amounts to $0.05 \mathrm{X}_{0}$ for the target and the tracking devices and to $0.54 \mathrm{X}_{0}$ for the PID.

The detection efficiency for electrons and positrons, studied using pair-production by $\gamma$-conversion in the detector material, is found to be very similar to photons except that it is shifted by $\approx 50 \mathrm{MeV}$ due to energy loss in the PID.

\footnotetext{
i) H. Müller et al., CERN Host Interface, User's manual, CERN/EP (1989).
} 


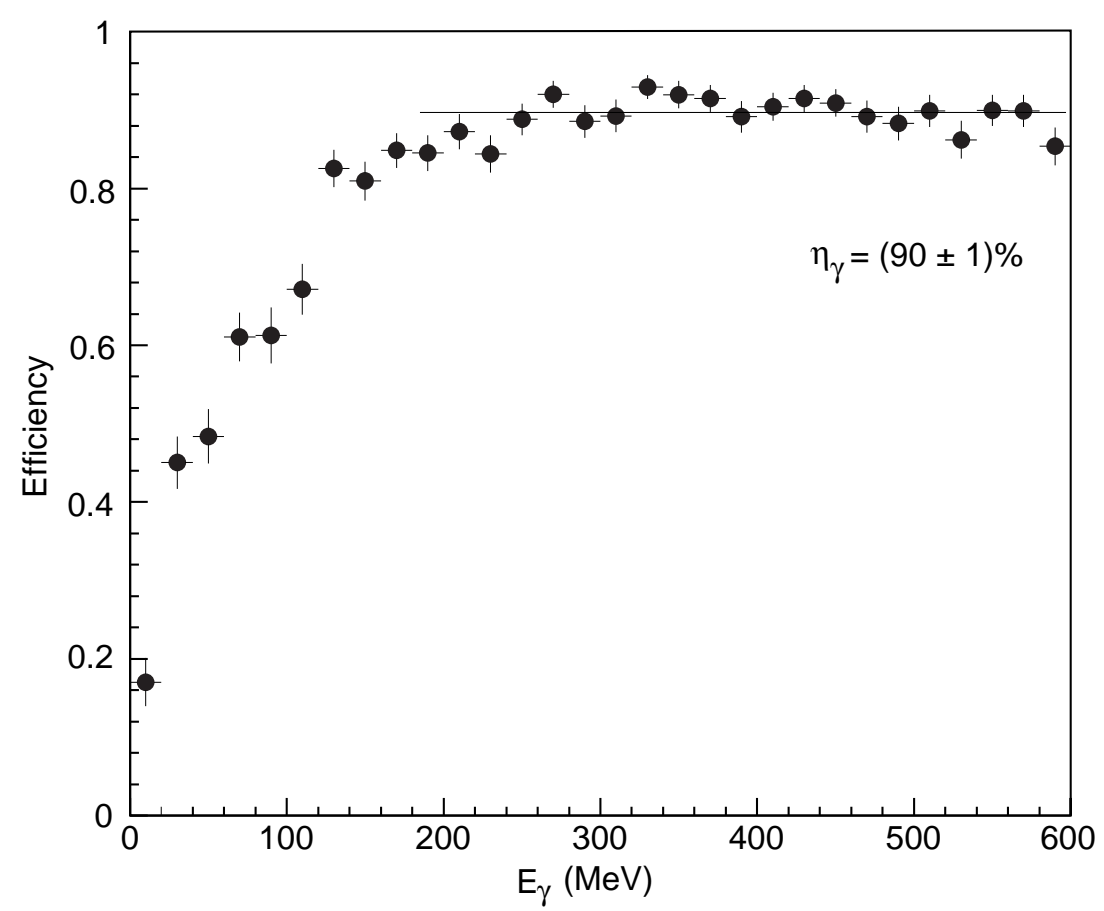

Figure 35: Photon detection efficiency as a function of the photon energy.

The spatial resolution for electromagnetic showers is deduced from the photon opening angle distribution in the decay of monoenergetic $(666 \mathrm{MeV}) \pi^{0}$ 's from the reaction given in (3). The measured distribution has been fitted with the expected distribution, with a minimum opening angle of $23^{\circ}$. The overall opening angle resolution for the two photons is unfolded and amounts to $\sigma_{\text {angle }}=7.9 \mathrm{mrad}$, which translates into a global conversion point resolution for one photon in space of $\sigma_{\text {space }}=4.5 \mathrm{~mm}$.

The spatial resolution of the calorimeter can also be studied by comparing the $r \phi$ - and $z$-coordinates of extrapolated charged tracks with the corresponding coordinates in the ECAL, determined by shower pattern recognition. A resolution of $\sigma=2.7 \mathrm{~mm}$ was found in the $r \phi$-plane after subtracting the contributions of multiple scattering $(2.2 \mathrm{~mm})$ and of measurement error in the tracking chambers $(0.8 \mathrm{~mm})$ to the track extrapolation uncertainty. In the $z$-direction a precise evaluation is not possible because of the large track extrapolation error $(>6 \mathrm{~mm})$ due to the DC measurement error dominating our resolution measurement.

Simulation studies show that in a sampling calorimeter the best energy resolution for photons with only a few hundred $\mathrm{MeV}$ energy is obtained by counting the number of track segments rather than measuring the energy deposited in the gas.

Figure 36 shows the hit multiplicities for tagged photons of a) $70 \pm 10 \mathrm{MeV}$ and b) $370 \pm$ $10 \mathrm{MeV}$ energy showering in the first layer of the calorimeter. The distribution is asymmetric for $70 \mathrm{MeV}$ due to the minimal shower requirement of three hits imposed by the shower pattern recognition algorithm. To parametrize the hit distributions, a dedicated function is constructed to account for the asymmetric shape (neither a Gaussian nor a Poisson function successfully fit the distributions):

$$
H(w, E)=N(E) \exp \left(\frac{-\left[w-w_{0}(E)\right]^{2}}{2 \sigma_{0}^{2}(E)+(1 / 2) \Omega(E)\left[\left(w-w_{0}\right)+\left|w-w_{0}\right|\right]}\right),
$$

where $w$ is the number of hits, and $w_{0}(E)$ is the hit multiplicity for which the function reaches the maximum value. The parameter $\Omega(E)$ takes account of the asymmetric shape. For photon energies $>150 \mathrm{MeV}$ the hit distribution is symmetric, $\Omega(E)$ vanishes and the function becomes a Gaussian with a variance equal to $\sigma_{0}^{2}(E)$. At lower energies, a good approximation is given by an effective variance $\sigma_{w}^{2}=\sigma_{0}^{2}(E)+2 \Omega^{2}(E)$. 

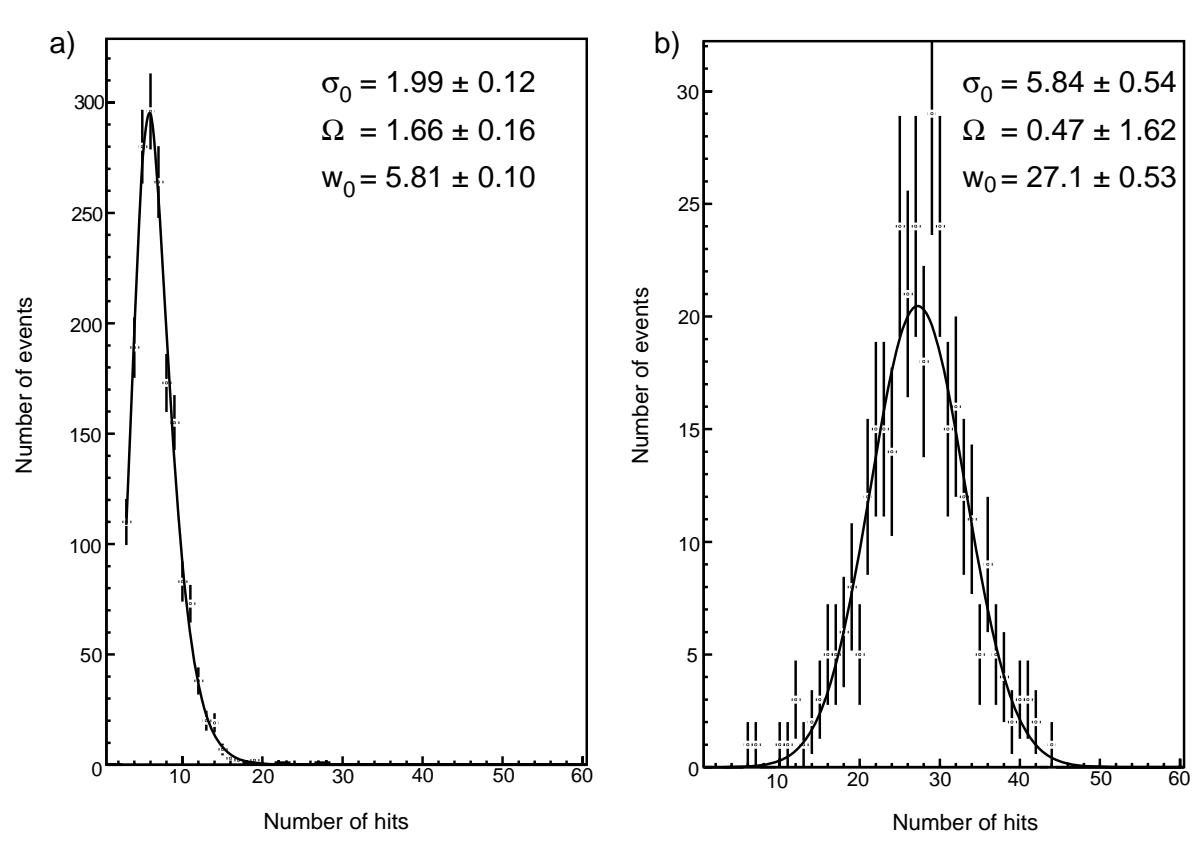

Figure 36: Distribution of the number of hit tubes in the calorimeter for two photon energies, a) $70 \mathrm{MeV}$ and b) $370 \mathrm{MeV}$.

In Fig. 37, the variation of the mean value $\langle w(E)\rangle$ is displayed as a function of the photon energy and of the layer in which the photon converted. The deviation of the data points from a straight line reflects the limited thickness of the calorimeter, leading to shower leakages, especially for photons converting in layers further out in a radial direction.

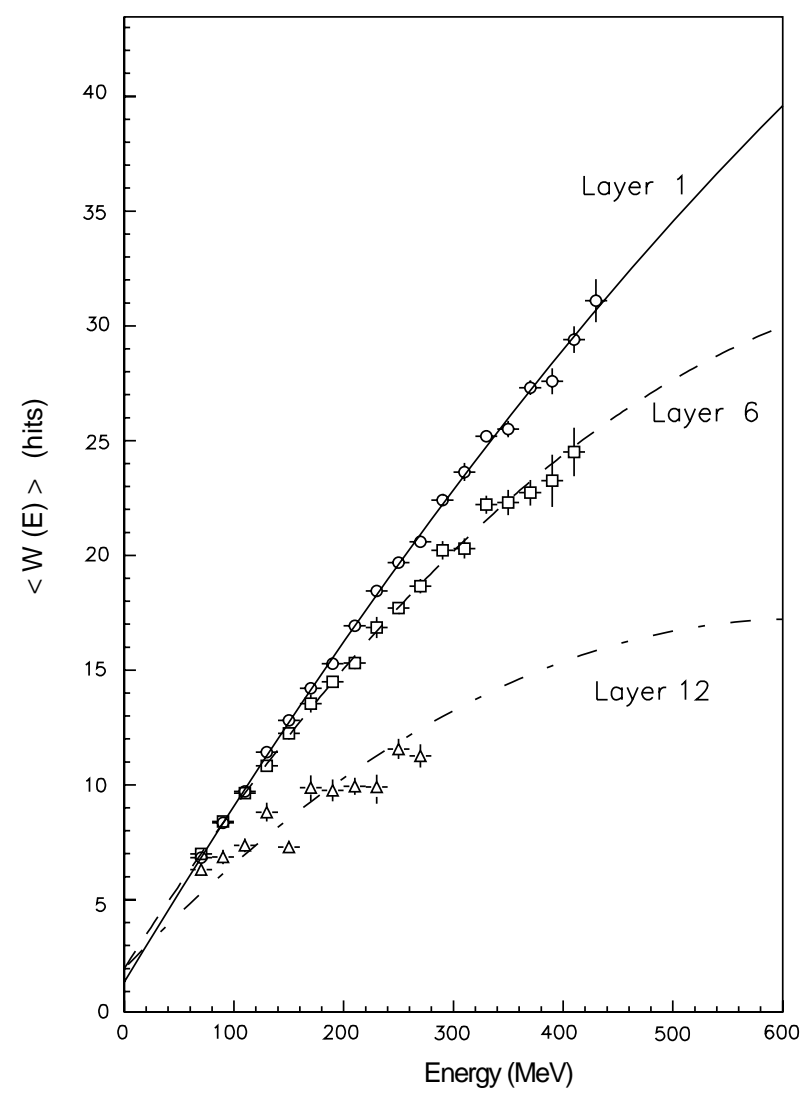

Figure 37: Mean value $\langle w(E)>$ of the hit distribution as a function of the photon energy for three conversion layers. 
The resolution of the hit distribution as a function of the photon energy depends only weakly on the conversion layer. The reduced resolution $R$, defined as

$$
\mathrm{R} \equiv \frac{\sigma_{w} \sqrt{E(\mathrm{GeV})}}{<w(E)>},
$$

is almost constant over a wide energy range, as shown in Fig. 38.
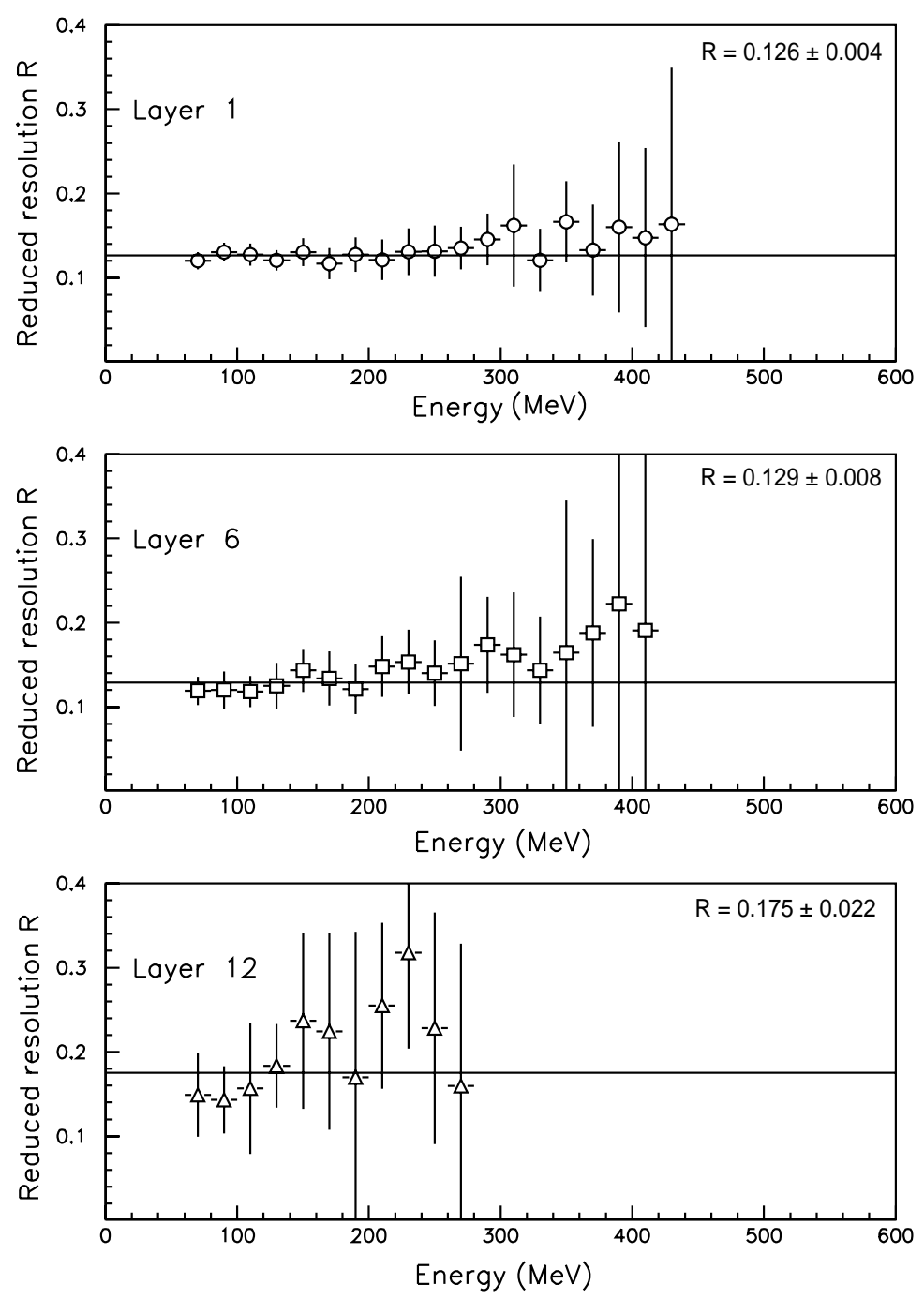

Figure 38: Reduced resolution (see text) as a function of the photon energy for three photon conversion layers at different depths.

The described performance of the ECAL enables the reconstruction of the $\mathrm{K}^{0}$ decay length with an average precision of $2 \mathrm{~cm}$ (FWHM) for the final state of two neutral pions $\mathrm{K}^{0} \rightarrow \pi^{0} \pi^{0} \rightarrow$ $4 \gamma$, where all the four photons have been detected. This translates into a resolution in the $\mathrm{K}^{0}$ decay time of $0.7 \tau_{\mathrm{S}}$. A detailed discussion of the analysis and results can be found in [25].

The distinction between electron and pion showers in the calorimeter was exploited for $\mathrm{e} / \boldsymbol{\pi}$ separation. The fine granularity allows for a precise determination of shower topologies. The neural network technique was applied for the separation, using the ECAL pattern information for the showers as input and relying exclusively on the wire hits because of their low-noise and high-efficiency performance. Calibration data from $\bar{p} p$ annihilation to four or six charged pions as well as converted photons $\left(\gamma \rightarrow \mathrm{e}^{+} \mathrm{e}^{-}\right)$were used to train the network. The purity of these samples was $99.7 \%$.

The ECAL pattern data were processed to yield the relevant information for presenting the shower characteristics to the neural network. The training was performed in the momentum 
range $200-600 \mathrm{MeV} / c$ with $50 \mathrm{MeV} / c$ bins. At lower momenta $(\leq 200 \mathrm{MeV} / c)$, pions do not reach the ECAL, thus allowing an obvious separation.

With the calibration data an efficiency of $75 \%$ in identifying electrons was achieved at $4 \%$ pion contamination over the entire momentum range (Fig. 39).
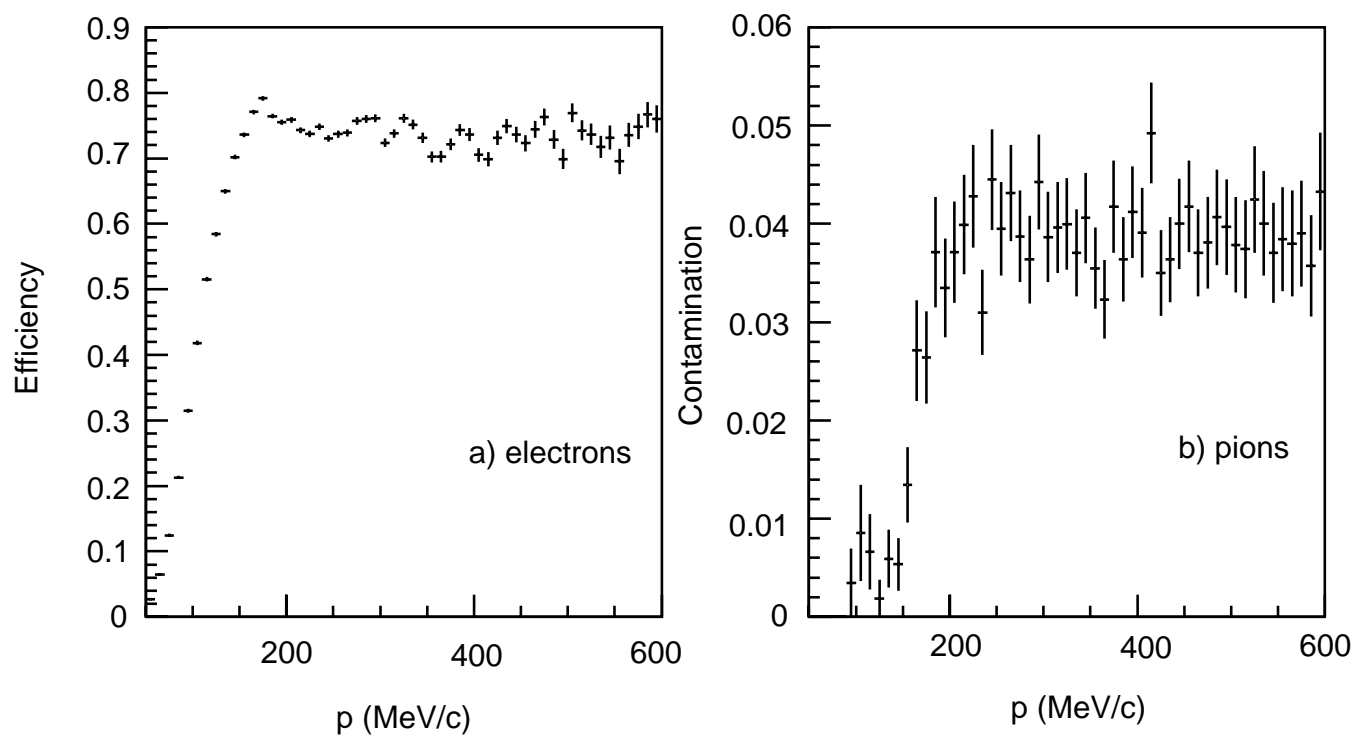

Figure 39: Electron identification efficiency a) and pion contamination fraction b) as a function of the momentum, using the showers in the calorimeter.

\section{The CPLEAR Trigger}

The small annihilation branching ratio for the desired channels of $\mathrm{K}^{0}\left(\overline{\mathrm{K}}^{0}\right)$ production $(1)$ and the high beam intensities require an intelligent and fast trigger system to select them from the unwanted background. This led to the implementation of a multilevel hardwired processor system (HWP), capable of utilizing the detector responses as soon as they become available for the selection process [2].

The trigger system aims at the best possible selection of $\mathrm{K}^{0}$ and $\overline{\mathrm{K}}^{0}$ in identifying a primary $\mathrm{K}^{ \pm} \pi^{\mp}$ pair and at the observation of the $\mathrm{K}^{0}\left(\overline{\mathrm{K}}^{0}\right)$ decay inside the fiducial volume of the detector. Nearly half of the produced neutral kaons $\left(96 \%\right.$ of the $\mathrm{K}_{\mathrm{L}}$ component) decay outside this volume.

The decisions are based on fast recognition of the charged kaon (using the PID hit maps), the number and topology of the charged tracks, the particle identification (using energy-loss, time-of-flight and Cherenkov light response) and kinematic constraints, as well as the number of showers in the ECAL.

Figure 40 shows the trigger decision steps in chronological order taking into account the availability in real time of the relevant detector information. The rate reductions produced by the different processor steps given in Fig. 40 depend on the particular detailed logic conditions set at each level.

The first decision step (Early Decision Logic - EDL) selects candidate events with at least two charged tracks (at least 2 hits in the inner scintillator S1), one of which must be a kaon, defined by the coincidence of the two scintillator layers and no Cherenkov response, i.e. S1 $\cdot \overline{\mathrm{C}} \cdot \mathrm{S} 2$. This first identification of charged kaons is further improved by requesting a minimum transverse momentum ( $p_{T}$ cut) in order to eliminate false kaon candidates due to slow pions.

The second step (Intermediate Decision Logic - IDL) identifies kaonic and other chargedtrack candidates using sectorized information (hit maps) from the tracking chambers in conjunction with the PID and classifies them depending on whether they originate inside ('primary track') or outside ('secondary track') the proportional chambers. 


\section{CPLEAR Fast Trigger}

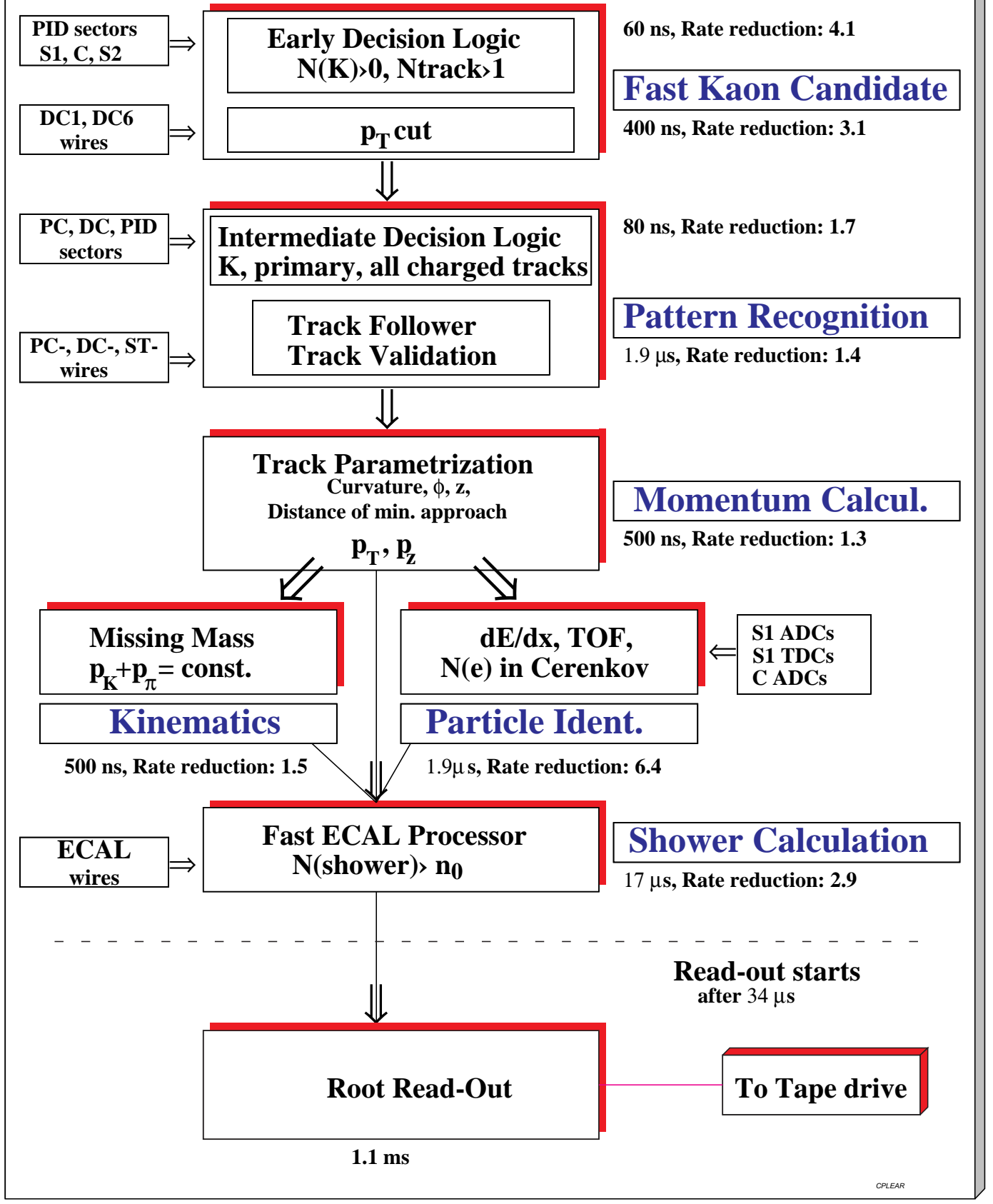

Figure 40: Logic and data flow diagram of the trigger system.

The third step (HWP1 processor) includes

- fine grain tracking and gathering of the front-end electronics digital information from the tracking chambers (Track Follower and Track Validation);

- track parametrization, to compute the track curvature $\rho$, the azimuthal angle $\phi$ at the point of minimum approach in the transverse plane, the distance of minimum approach $\epsilon$, and the derivation of the $z$-coordinate from the streamer tubes;

- calculation of transverse and longitudinal momenta of the 'primary' particles and application of a kinematical cut on a minimum value of the sum $p_{\mathrm{K}}+p_{\pi}$ (equivalent to the missing mass) for all $\mathrm{K} \pi$-pairs;

- the charge balance $\left(\sum Q_{i}=0\right)$ required for events with an even number of tracks (2 or 4$)$. 
The quality of the track-finding and parametrization algorithm can be seen from Fig. 41, where the track curvature, $\rho$, computed by the trigger processor HWP1 is plotted as a function of the curvature measured offline for 2000 events.

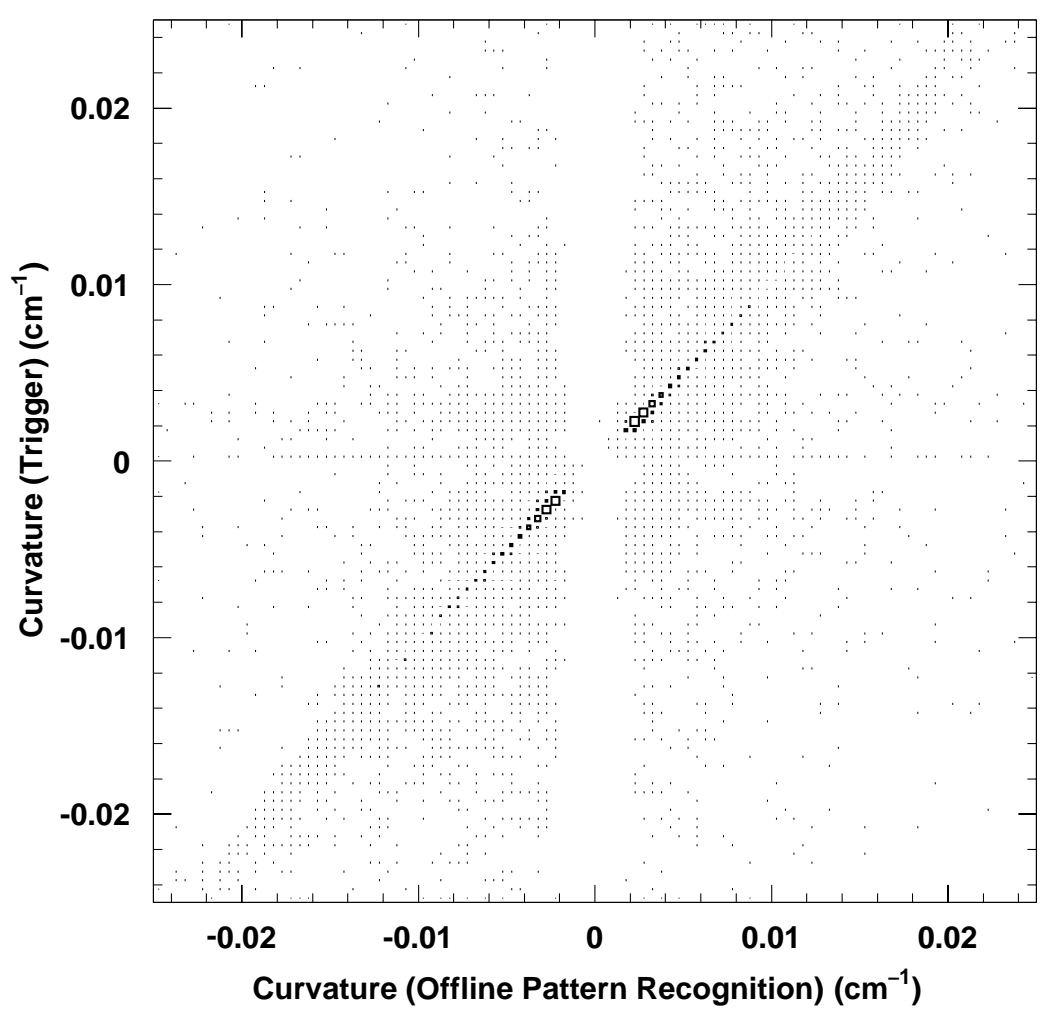

Figure 41: Online versus offline track curvature.

The next step (HWP2 processor) improves the $\mathrm{K}^{ \pm} \pi^{\mp}$-pair identification by comparing the measured energy-loss and Cherenkov response for the kaon(s) as well as the TOF difference $\Delta t=t_{\mathrm{K}}-t_{\pi}$ to their expected values, according to the previously determined track parameters.

For candidate 'neutral' events, i.e. events with only two primary $(\mathrm{K} \pi)$ tracks, the showers in the ECAL are quickly identified by a dedicated processor (HWP2.5). The HWP2.5 processor examines the ECAL hits and determines the number of showers using an algorithm acting on the hit group pattern information in the $r \phi$-plane. This has proved to be the most powerful method for the identification of electromagnetic showers, given the low energy of the photons. There is no distinction between charged and neutral showers. The requirement for a minimum number of showers, $n_{0}=5$ or 6 , eliminates all $\mathrm{K}_{\mathrm{L}}$ events decaying outside the fiducial volume of the detector.

The overall rejection factor of the trigger is $\approx 10^{3}$, allowing a read-out rate of $\approx 450$ events per second at an average beam rate of $800 \mathrm{kHz}$. The global acceptance for $\mathrm{K}^{0}, \overline{\mathrm{K}}^{0}$ produced in reaction (1) and decaying in the fiducial volume of the detector is $\approx 7 \%$, mainly determined by geometrical factors.

The decision time of each stage varies of the trigger from $60 \mathrm{~ns}$ (EDL) to $17 \mu \mathrm{s}$ (HWP2.5). The read-out strobe is delivered to front-end electronics $34 \mu$ s after beam counter signal arrival.

The trigger processors have been constructed using custom ECL electronics (VME standard [26]) . An internal system clock is used to synchronize the data flow and logic decisions, starting with the fine grain tracking in HWP1.

The communication and data flow between the trigger processors and the detector electronics benefits from the read-out sectorization in the transverse plane, i.e. 32 PID sectors and 64 tracking chamber mini-sectors. Data requested by the processors using the appropriate mini-sector address are returned from the front-end electronics hardwired processor interfaces together with the information requested. This form of dialogue is faster than using a handshake 
procedure.

Look-up tables are used whenever the trigger processors request online calibrated frontend data and for the numerical computation of physical quantities, in particular for the track parametrization stage and for the HWP2 processor.

The multilevel processor system is controlled using a pipelined logic (sequencer) unit. A trigger control system provides all the signals required by the detector front-end electronics and initiates the transfer of information to the Root Read-out system. It is capable of rejecting a wrong candidate event as soon as it is possible following its identification. After a 'clear time' of $350 \mathrm{~ns}$ the trigger system is ready to accept the next candidate event. This minimizes the trigger dead-time due to the large number of rejected events. The overall dead-time of the trigger is about $25 \%$ at a $1 \mathrm{MHz}$ antiproton rate.

The performance and stability of the trigger system are monitored online using data gathered by a dedicated interface called 'SPY'. This collects the data input to the processors (the hit maps and front-end data from the detectors) and all intermediate and final data and logic decisions generated by the trigger system.

By using the SPY data and the results of a full trigger simulation, which includes a simulation of the digitization for all processors, the functionality and the efficiency of each stage can be measured. For each stage, the functionality is determined by comparing the decision of the trigger with the decision computed by the trigger simulation using the input data to this particular stage as read by the SPY. The functionality reflects the behaviour of a particular trigger stage, including internal timing imperfections. For all stages, the functionality is better than $98 \%$. To determine the efficiency of each stage, the input information of the stage used by the simulation is re-evaluated using the Root Read-out data of each sub-detector. The efficiency therefore depends not only on the functionality but also on the quality and timing imperfections of the data transfer between the sub-detector front-end electronics and the trigger. During data taking, the efficiencies are measured each hour on a data sample of 2000 events and the functionality checks are performed once a day. The overall trigger efficiency, continuously measured during the run, is around $88 \%$ with variations at a level of $2 \%$.

\section{$7 \quad$ Summary}

The CPLEAR detector has been fully operational since 1992. All its subdetectors beam monitors, tracking devices, particle identification detector, electromagnetic calorimeter have achieved their expected performance. Specially designed hardwired processors have been successfully implemented to trigger the experiment and remove the unwanted background. The detector has run smoothly during the last 5 years and accumulated nearly $2 \times 10^{8}$ decays of strangeness-tagged neutral kaons entering our final data sample, allowing precision studies of CP-, T- and CPT-symmetries [27].

\section{Acknowledgments}

We acknowledge the important contribution made by the PPE technical support group (G. Muratori and M. Price). We thank D. Fromm and H. Rigoni for their help with the gas distribution system, and C. Millerin and his team for their assistance in the electronics production.

This work was supported by the following institutions: the French CNRS/Institut National de Physique Nucléaire et de Physique des Particules, the French Commissariat à 1'Energie Atomique, the Greek General Secretariat of Research and Technology, the Netherlands Foundation for Fundamental Reserach on Matter (FOM), the Portuguese JNICT and INIC, the Ministry of Science and Technology of the Republic of Slovenia, the Swedish Natural Science Research Council, the Swiss National Science Foundation, the UK Particle Physics and Astronomy Research Council (PPARC), and the US National Science Foundation. 


\section{References}

[1] E. Gabathuler and P. Pavlopoulos, Strong and Weak CP violation at LEAR, Proc. Workshop on Physics at LEAR with Low Energy Cooled Antiprotons, Erice, 1982, eds. U. Gastaldi and R. Klapisch (Plenum, New York, 1984) 747.

[2] D. Tröster et al., Nucl. Instrum. Methods A279 (1989) 285.

[3] C.P. Bee et al., The CPLEAR data acquisition system, Proc. CHEP92 Conference, CERN 92-07 (1992) 293;

C.P. Bee et al., The CPLEAR data acquisition system, in preparation.

[4] M. Van den Putte et al., IEEE Trans. Nucl. Sci. 37 (1990) 53.

[5] M. Van den Putte, Ph.D. thesis, Delft University of Technology, Netherlands (1994).

[6] J. De Vries, Ph.D. thesis, Delft University of Technology, Netherlands (1987);

R.W. Hollander et al., Materials Science Forum 105-110 (1992) 1883.

[7] Ch. Bula, Ph.D. thesis 9808, Swiss Federal Institute of Technology, Zürich, Switzerland (1992).

[8] M. Dejardin et al., Nucl. Instrum. Methods A283 (1989) 484.

[9] M. Dejardin, Ph.D. thesis, University of Paris VII, France (1992).

[10] P.M. Sanders, Ph.D. thesis, University of Liverpool, UK (1991).

[11] J.M. Bennet et al., A Streamer Tube detector for operation at high rates in the CPLEAR experiment at CERN, Nucl. Instrum. Methods, in print.

[12] D.M. Lee et al., Nucl. Instrum. Methods A256 (1987) 329.

[13] M. Dodgson, Ph.D. thesis, University of Liverpool, UK (1990).

[14] R. Rickenbach et al., Nucl. Instrum. Methods A279 (1989) 305.

[15] A. Angelopoulos et al., Nucl. Instrum. Methods A311 (1992) 79.

[16] 3M Fluorinert Product Information (1989).

[17] M. Danielsson and H.-J. Gerber, Nucl. Instrum. Methods A334 (1993) 339.

[18] A. Chlopik et al., Nucl. Instrum. Methods A301 (1991) 424.

[19] W.E. Earle et al., Nucl. Instrum. Methods A333 (1993) 513.

[20] M. Dejardin, CPLEAR note CP/SW/043 (1993).

[21] R. Adler et al., The CPLEAR electromagnetic calorimeter, in preparation.

[22] P. Bloch et al., Nucl. Instrum. Methods A297 (1990) 126.

[23] R. Adler et al., Nucl. Instrum. Methods A321 (1992) 458.

[24] F. Bal et al., Nucl. Instrum. Methods A323 (1992) 511.

[25] B. Pagels et al., Reconstruction of a particle decay containing intermediate decays of invisible neutrals, ETHZ-IPP Internal Report 95-4, 1995.

[26] D.A. Tröster et al., CPLEAR note CP/HWP/004, 1990.

[27] R. Adler et al., Measurement of the $\mathrm{K}_{\mathrm{L}}-\mathrm{K}_{\mathrm{S}}$ mass difference using semileptonic decays of tagged neutral kaons, Phys. Lett. B363 (1995) 237;

R. Adler et al., Measurement of the CP violation parameter $\eta_{+-}$using tagged $\mathrm{K}^{0}$ and $\overline{\mathrm{K}}^{0}$, Phys. Lett. B363 (1995) 243;

R. Adler et al., First observation of a particle-antiparticle asymmetry in the decay of neutral kaons into $\pi^{0} \pi^{0}$, Z. Phys. C70 (1996) 211;

R. Adler et al., Search for CP violation in the decay of neutral kaons to $\pi^{+} \pi^{-} \pi^{0}$, Phys. Lett. B370 (1996) 167. 